\title{
Nonlinear and nonstationary influences of geomagnetic activity on the winter North Atlantic Oscillation
}

\author{
Yun Li, ${ }^{1}$ Hua Lu, ${ }^{2}$ Martin J. Jarvis, ${ }^{2}$ Mark A. Clilverd, ${ }^{2}$ and Bryson Bates ${ }^{3}$ \\ Received 17 February 2011; revised 11 May 2011; accepted 18 May 2011; published 19 August 2011.
}

[1] The relationship between the geomagnetic $a a$ index and the winter North Atlantic

Oscillation (NAO) has previously been found to be nonstationary, being weakly negative during the early 20 th century and significantly positive since the 1970s. The study reported here applies a statistical method called the generalized additive modeling (GAM) to elucidate the underlying physical reasons. We find that the relationship between $a a$ index and the NAO during the Northern Hemispheric winter is generally nonlinear and can be described by a concave shape with a negative relation for small to medium $a a$ and a positive relation for medium to large $a a$. The nonstationary character of the $a a-\mathrm{NAO}$ relationship may be ascribed to two factors. First, it is modulated by the multidecadal variation of solar activity. This solar modulation is indicated by significant change points of the trends of solar indices around the beginning of solar cycle 14, 20, and 22 (i.e., $\sim 1902 / 1903, \sim 1962 / 1963$, and 1995/1996). Coherent changes of the trend in the winter time NAO followed the solar trend changes a few years later. Second, the $a a$-NAO relationship is dominated by the $a a$ data from the declining phase of even-numbered solar cycles, implying that the 27 day recurrent solar wind streams may be responsible for the observed $a a-\mathrm{NAO}$ relationship. It is possible that an increase of long-duration recurrent solar wind streams from high-latitude coronal holes during solar cycles 20 and 22 may partially account for the significant positive $a a$-NAO relationship during the last 30 years of the 20th century.

Citation: Li, Y., H. Lu, M. J. Jarvis, M. A. Clilverd, and B. Bates (2011), Nonlinear and nonstationary influences of geomagnetic activity on the winter North Atlantic Oscillation, J. Geophys. Res., 116, D16109, doi:10.1029/2011JD015822.

\section{Introduction}

[2] The North Atlantic Oscillation (NAO) is a dominant mode of broad-scale climate variability in the Northern Hemisphere (NH) [Hurrell, 1995; Hurrell et al., 2003]. Being most active during winter months, it is directly linked to the variation of westerly winds, orchestrates the strength and orientation of storm tracks, and dictates precipitation levels and fluctuations in marine ecosystems over the Atlantic as well as the adjacent regions [Stephenson et al., 2000]. Positive NAO is associated with a net displacement of air from the Arctic and Icelandic regions toward the subtropic belt near the Azores and the Iberian Peninsula by strengthening westerly winds over the North Atlantic Ocean. Stronger westerlies bring more warm moist air to the European continent and gives rise to milder maritime winters. Negative NAO is associated with an opposite redistribution of air mass between the Arctic and the subtropical Atlantic

\footnotetext{
${ }^{1}$ CSIRO Climate Adaptation Flagship, CSIRO Mathematics, Informatics and Statistics, Wembley, Western Australia, Australia.

${ }^{2}$ British Antarctic Survey, Cambridge, UK.

${ }^{3}$ CSIRO Climate Adaptation Flagship, CSIRO Marine and Atmospheric Research, Wembley, Western Australia, Australia.

Copyright 2011 by the American Geophysical Union. 0148-0227/11/2011JD015822
}

with weaker westerlies over the North Atlantic. It corresponds to colder than normal European winters. It is critical to understand the mechanisms that control and affect the $\mathrm{NAO}$ and its temporal evolution as it is associated with large variations in weather and climate over much of the globe on interannual and longer time scales [Hurrell and Deser, 2010].

[3] Despite its importance, the fundamental mechanisms behind the variability of the NAO remain in debate [Hurrell and Deser, 2010; Saenger et al., 2009]. It has been suggested that external forces might nudge the NAO to remain in either its positive or negative phase over an extended period. Observations have shown that the NAO may be modulated by a multidecadal oscillation in Atlantic sea surface temperature [Enfield et al., 2001; Gray et al., 2004; Knight et al., 2006]. Low-frequency variations of the NAO were previously attributed to perturbations associated with the thermal storage effect of the ocean, sea ice, or snow [Gong et al., 2003; Hall and Visbeck, 2002; Mehta et al., 2000; Rodwell et al., 1999], or to anthropogenic forcing and/or ozone depletion in the stratosphere [Corti et al., 1999; Gillett et al., 2003; Shindell et al., 1999; Woollings et al., 2010a]. Studies based on those proposed mechanisms have been carried out using general circulation models (GCMs) [Gong et al., 2002; Hoerling et al., 2001]. However, the simulated NAOs often exhibit little multidecadal variation compared with observa- 
tions [Osborn, 2004]. In particular, it remains unclear what has caused the enhanced interannual variability of the NAO over the last half of the 20th century and a more positive trend of the NAO in 1970s-1990s [Feldstein, 2002; Hurrell and Deser, 2010].

[4] It has also been suggested that changes in solar and geomagnetic activity may modulate the NAO [Bochniček and Hejda, 2005; Kodera, 2002, 2003]. Kodera [2002] and Ogi et al. [2003] found that in winter the spatial and temporal structure of the NAO is greatly modified by the 11 year solar cycle (SC). Stronger and more significant correlations were found to exist between geomagnetic activity and the NAO than with the 11 year SC [Bochnićek and Hejda, 2005; Thejll et al., 2003]. Boberg and Lundstedt [2002, 2003] found that the variation of the winter NAO is correlated with the electric field strength of the solar wind, and suggested a solar wind generated electromagnetic disturbance in the ionosphere may dynamically propagate downward to the stratosphere and influence surface NAO. Changes of temperature near the Atlantic basin have also been statistically linked to geomagnetic perturbations [Seppälä et al., 2009]. Woollings et al. [2010b] showed that a stronger signal in the eastern part of the North Atlantic was associated with solar wind driven open solar flux when compared with that associated with the F10.7 cm solar flux. Lu et al. [2008b] have shown that there is a robust relationship between solar wind dynamic pressure and the Northern Annular Mode (NAM), which resembles the behavior of the NAO near the surface. They showed that stratospheric wind and temperature variations are positively projected onto the NAM when the 11 year $\mathrm{SC}$ is at its maximum phase during winter, and negatively projected onto the NAM during the 11 year SC minimum phase in spring. Paluš and Novotná [2007] found that there were common oscillatory modes in the geomagnetic $a a$ index, the NAO and surface temperature. It has been also suggested that solar wind induced geomagnetic activity may alter stratospheric chemistry through particle precipitation and descent of odd Nitrogen $\left(\mathrm{NO}_{\mathrm{x}}\right)$ [Randall et al., 2005, 2006, 2007; Solomon et al., 1982]. Other studies suggested that solar wind induced geomagnetic activity may perturb atmospheric circulation dynamically through a change in planetary wave reflection conditions [Arnold and Robinson, 2001; Lu et al., 2008a].

[5] It has also been shown that solar activity may indirectly alter cloud cover via a modulation of the galactic cosmic ray (GCR) flux [e.g., Pudovkin and Veretenenko, 1995, 1996; Svensmark and Friis-Christensen, 1997; Svensmark et al., 2009; Harrison and Stephenson, 2006], while others found the GCR-cloud connection is rather weak [Laken et al., 2009; Pierce and Adams, 2009]. Changes in solar wind electric fields may also perturb the global electrical circuit, which extends into the lower atmosphere at high magnetic latitudes, and consequently precipitation and cloud cover in the fair weather part of the global circuit [Tinsley et al., 2007; Kniveton et al., 2008]. Since the solar wind driven geomagnetic activity also modulates the cosmic ray flux, it has been suggested that it may affect the amount of tropospheric ionization and cloud cover [Shaviv, 2005].

[6] While those statistical correlations suggest there might be a causal link between solar wind driven geomagnetic activity and the NAO, a sound mechanism is required for better understanding and to rule out coincidental correlations. What is most puzzling is that the relationship between geomagnetic activity and the NAO appears to be nonstationary, being significantly positive since the 1970 s and weakly negative in the early part of the 20th century [Bucha and Bucha, 1998; Palamara and Bryant, 2004]. Paluš and Novotná [2009] found that the phase coherence between the geomagnetic $a a$ index and the NAO was statistically significant only from the 1950s onwards while no significant phase coherence was found in the earlier period. The nonstationary behavior of the $a a-\mathrm{NAO}$ relationship was attributed to the much lower geomagnetic activity occurring in the early part of the 20th century than in 1950-2000 which led to a significant shift of atmospheric circulation patterns [Bucha and Bucha, 1998]. It has also been suggested that the nonstationary behavior of the $a a-\mathrm{NAO}$ relationship might be due to a multidecadal scale modulation of the geomagnetic NAO relationship [Thejll et al., 2003].

[7] Geomagnetic activity is primarily driven by the solar wind which encounters the Earth's magnetic field at the magnetopause. Solar wind is emitted from the Sun either as high-speed wind streams from corotating interactive regions (CIRs) of coronal holes, transient wind streams associated with coronal mass ejections (CMEs), or slow solar winds from the borders of coronal holes [Emery et al., 2009]. Highspeed solar wind generated from CIRs tends to recur with a $\sim 27$ day periodicity and is most prominent in the descending phases of SCs. It has been noticed that the characteristics of the $\sim 27$ day periodicity in the geomagnetic $a a$ index are different during the decay phases of even- and odd-numbered SCs [Cliver et al., 1996]. Studies have shown that recurrent activity was stronger following even-number maxima [Bumba and Hejna, 1991; Hapgood, 1993; Rangarajan, 1991] and prominent 27 day recurrent geomagnetic activity tends to persist longer during the decline of even-numbered SCs than in odd-numbered ones [Sargent, 1985, 1986]. If, as postulated, geomagnetic activity has an impact on the NAO and near surface climate, the 27 day recurrence events may have a detectable effect on the interannual and longer-term variations of the NAO. Thus, a step toward examining such a possibility would be to examine whether or not the $a a-\mathrm{NAO}$ relationship becomes more distinct during the declining phase of even-numbered SCs.

[8] In this study, we reexamine the $a a-\mathrm{NAO}$ relationship in order to elucidate the underlying physical reasons for its nonstationary behavior. Like previous studies, we focus on the most dynamically active season, i.e., the extended $\mathrm{NH}$ winter period from December to March for the period of 1868-2009. We first apply a statistical method called generalized additive modeling (GAM) [Wood, 2006, 2008] to search for statistically significant linear and/or nonlinear relationships between the $a a$ index and the NAO for the period of 1868-2009. We then use the sequential MannKendall (SMK) test [Gerstengarbe and Werner, 1999] to study the multidecadal variation of the NAO and its possible link to the change points in the trends of solar and geomagnetic indices. We investigate the nonstationary behavior of the $a a-\mathrm{NAO}$ relationship by building nonlinear and linear regression models using the GAM technique for each subperiod separated by two nearby change point years that are identified by the SMK test. In order to examine whether or 
not the recurrent solar wind events play a role in determining the $a a$-NAO relationship, the linear models and GAMs are also built based on the data grouped according to the ascending/declining phases of odd/even number SCs.

\section{Data}

[9] The NAO time series used here is the normalized difference between the sea level atmospheric pressures at Gibraltar and southwest Iceland. The monthly pressure at each location is normalized by subtracting the mean and dividing by the standard deviation. A standard period (19511980), instead of the whole recording period, is considered for estimating the mean and the standard deviation [Jones et al., 1997]. Monthly NAO indices since 1821 have been downloaded from the Website of Climate Research Unit of the University of East Anglia, UK (http://www.cru.uea.ac. uk). Due to the large amount of missing data before 1824, the time series of December-March mean (denoted as $\mathrm{NAO}_{\mathrm{DJFM}}$ hereafter) used here only covers the period of 1825-2009. The year of NAO $\mathrm{DJFM}_{\text {Is }}$ is defined by the year in January. That is, $1825 \mathrm{NAO}_{\text {DJFM }}$ represents the mean NAO value for the $1824 / 1825$ winter and so forth.

[10] The geomagnetic aa index [Mayaud, 1972] is commonly used to monitor the reactions of Earth's upper atmosphere (e.g., magnetosphere and ionosphere) and magnetic field to changes in the incoming solar wind. The monthly $a a$ index from 1 January 1868 to 31 December 2009 is used here. Its winter averages were calculated in the same way as that used for $\mathrm{NAO}_{\text {DJFM }}$ and are denoted as $a a_{\text {DJFM }}$ hereafter.

[11] Three different solar input data sets are used to study multidecadal variation of solar activity and its possible modulation effect on the $a a-\mathrm{NAO}$ relationship. The first one is the sunspot number $\left(\mathrm{R}_{\mathrm{z}}\right)$, a commonly used proxy for the phase of the given SC at century time scales. It is also a proxy for solar electromagnetic radiation which creates and maintains the Earth's ionosphere and the tidal winds in the atmosphere. Monthly international sunspot number data from January 1749 to December 2009 are used here; they are available at http://www.ngdc.noaa.gov/. The second and third solar data sets are solar sunspot area in the Sun's northern hemisphere (NSSA) and the solar north-south asymmetry index $A s=(N-S) /(N+S)$ where $N$ and $S$ are the total sunspot area in the solar northern and southern hemispheres, respectively. The asymmetry index $A s$ is characterized by the time dependence of various activity indices in two hemispheres of the Sun which often display mismatch in both phase and power spectra [e.g., Li et al., 2002]. The reason we extend our analysis to include $A s$ here is because many studies have shown that it represents a fundamental characteristic of solar activity, which is controlled by solar magnetic fields instead of by the 11 year solar cycle [Badalyan and Obridko, 2011, and references therein]. Monthly sunspot area and its northsouth asymmetry are available at http://solarscience.msfc. nasa.gov/greenwch.shtml; this data set contains measurements collected by the Royal Greenwich Observatory (May 1874 to December 1975) and by the U.S. Air Force (1976 to present). The mismatch between the two different instruments pre-1975 and after 1975 was calibrated by Dr. David Hathaway at NOAA/NASA by using the additional data from the Mount Wilson photographic plate collection from
1917 to 1982 . The December-March mean NSSA and As from the period of 1874-2009 are used here.

\section{Methods}

[12] For the first time, a nonparametric regression method called Generalized Additive Model (GAM) [e.g., Gu, 1992; Hastie and Tibshirani, 1990; Wood, 2006, 2008] is used to build statistical relationships between the winter NAO and the geomagnetic $a a$ index. GAM is a statistical modeling framework which blends a generalized linear model (GLM) with additive terms, in which some of the terms in the model are fitted as smooth, nonlinear functions of the explanatory variables. Rather than prespecifying the form of the function, the data are used to estimate the shape of smooth functions. This provides a more flexible modeling approach (than GLM) which allows the users to explore the nonlinear relationship between the response and the explanatory variables. GAM has been used extensively in the analysis of air pollution, health, environment and ecology [e.g., He et al., 2006; Liu et al., 2009; McLeod and Pople, 2010; Mikolajczyk et al., 2010] due to its flexibility to model the relationship between the predictants and predictors. More recently, GAMs have been applied to hydrological and climatic research areas [e.g., Cox et al., 2005; Mestre and Hallegatte, 2009; Morton and Henderson, 2008]. A brief description of GAM is given in Appendix A.

[13] The SMK test [Gerstengarbe and Werner, 1999] is employed to detect the approximate times when a significant change of trend occurs in a given time series. It is a nonparametric test and comes under the class of rank tests and has been frequently used to detect approximate potential trend change points in a time series [Sneyers, 1990; Taubenheim, 1989]. For a given sampling series, the test is carried out by ranking the progressive and retrograde score series of this sample. The null hypothesis is that there is no turning point in the trend of the sampled time series under investigation. In order to prove or to disprove the null hypothesis, the statistical procedure introduced by Gerstengarbe and Werner [1999] is used. See Appendix B for mathematical details. As the method can only detect the approximate time when a change of the trend may occur, we find that it is often the case that multiple changes can be detected over a rather short period. As our focus is on multidecadal variation, in the case where multiple change points are detected within 11 years, we choose the change point with the largest confidence level at the time when the trend has changed. In such cases, the trend change may actually take a few years in the real world.

[14] The regression modeling based GAM is first carried out for the entire period of 1868/1869 winter to 2008/2009 winter, during which monthly $a a$ are available. The same analysis is also carried out for the subperiods separated by change points that are identified by the SMK test and by grouping the data into odd/even-numbered SCs and/or ascending/declining phases of the SCs.

\section{Results}

\subsection{Nonlinear $a a-N A O$ Relationship}

[15] Figure 1a shows the GAM fit of $\mathrm{NAO}_{\mathrm{DJFM}}$ and $a a_{\text {DJFM }}$ from 1869 to 2009 (where the years are defined by 

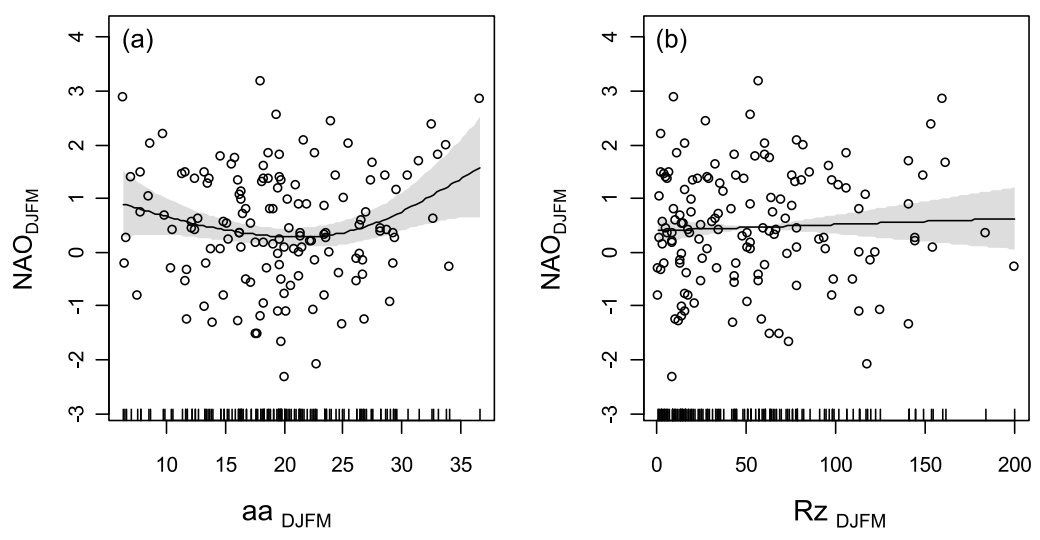

Figure 1. (a) Scatterplot of the December-March mean $\mathrm{NAO}_{\mathrm{DJFM}}$ against the December-March mean geomagnetic $a a$ index $a a_{\text {DJFM }}$ from the period of 1869-2009. (b) Scatterplot of NAO DJFM against the December-March mean sunspot number $\mathrm{R} \mathrm{z}_{\mathrm{DJFM}}$ from the period of 1825-2009. The black lines denote the GAM fitting while the shaded region shows the $95 \%$ confidence interval for GAM model. When the output of the GAM is linear, only the linear model and its $95 \%$ confidence interval are shown. The small vertical bars inside of the $x$ axis represent the corresponding values of $\mathrm{Rz}_{\mathrm{DJFM}}$ and $a a_{\mathrm{DJFM}}$ for all the samples.

the January date). The smooth function $f$ (see Appendix A) of the GAM fitting with an effective degree of freedom (EDF) equal to 2.5 (significant at the 0.05 level) indicates that there is statistical evidence of a nonlinear relationship between $\mathrm{NAO}_{\mathrm{DJFM}}$ and $a a_{\text {DJFM }}$ for the period of 1869 2009. Table 1 suggests that about $8 \%$ of the variance in $\mathrm{NAO}_{\text {DJFM }}$ may be explained by $a a_{\text {DJFM }}$. This $a a-\mathrm{NAO}$ relationship is marked by a nonlinear concave shape in Figure 1a with a negative relation for small to medium $a a$ and a positive relation for medium to large $a a$. No significant linear model can be built between $a a_{\text {DJFM }}$ and NAO DJFM (see Table 1). This result suggests that a nonlinear relationship is statistically superior to the linear one for the entire period of 1869-2009. This is not only because the Akaike information criterion (AIC) [Akaike, 1974] of nonlinear models is much lower compared to the corresponding AIC of the linear model (LM), but also because it is evident through the larger value of $\mathrm{R}^{2}$.

[16] On the other hand, no significant Rz-NAO relationship can be established by either linear or GAM models either for the period of 1825-2009 during which both the NAO DJFM and $\mathrm{Rz}_{\text {DJFM }}$ were available (see Figure $1 \mathrm{~b}$ ). No significant linear or nonlinear Rz-NAO relationship can be established of the shorter common period of 1869-2009 either (not shown). Thus, at the interannual time scale, solar wind driven geomagnetic activity, rather than solar electromagnetic radiation or solar irradiance, is more likely to directly affect the winter NAO.

\subsection{Multidecadal Modulation by Solar Activity}

[17] Figure 2a shows the time series of $\mathrm{NAO}_{\mathrm{DJFM}}$ from 1825 to 2009 . Figure $2 \mathrm{~b}$ shows the progressive $(U(t)$, the solid curve) and retrograde $(V(t)$, the dashed curve) scores of $\mathrm{NAO}_{\text {DJFM }}$ calculated by the SMK test. $U(t)$ and $V(t)$ cross each other around the winters 1907/1908, 1970/1971, and $1995 / 1996$ (shown as vertical red dashed lines) where either $U(t)$ and $V(t)$ reaches or exceeds the confidence limits of $95 \%$ (horizontal blue dashed lines) before or after these crossing points. The crossing points indicate that a change in multidecadal trend occurred at those times. Figure $2 \mathrm{~b}$ shows that there was no significant change in $\mathrm{NAO}_{\mathrm{DJFM}}$ trend before 1907/1908. From 1908, NAO DJFM $_{\text {started to decrease }}$ until 1970/1971 and then increase until 1995/1996. Since then, $U(t)$ and $V(t)$ cross each other frequently, implying a change of $\mathrm{NAO}_{\mathrm{DJFM}}$ trend might have taken place over an extended period from 1996 to 2009. It may also suggest that the $\mathrm{NAO}_{\text {DJFM }}$ trend has started to behave in a similar way to that in 1825-1880 during which period no significant trend was presented.

[18] Figure $2 \mathrm{c}$ shows the time series of $a a_{\text {DJFM }}$ from 1869 to 2009 . Figure $2 \mathrm{~d}$ shows that its progressive $U(t)$ and retrograde $V(t)$ scores do not cross each other at any time for the entire period, implying that no significant change of trend in $a a_{\text {DJFM }}$ can be detected by SMK. A similar result is obtainable if the annual mean $a a$ is used (not shown). Thus, over the extended period of 1869-2009, the long-term trend of the $\mathrm{NAO}$ is not statistically related to that of geomagnetic activity.

Table 1. Estimated Coefficients by Fitting Linear and GAM Models to the Relationship Between $a a_{\text {DJFM }}$ and NAO DJFM $_{\text {for the Entire }}$ Period of $1869-2009^{\mathrm{a}}$

\begin{tabular}{|c|c|c|c|c|c|c|c|c|}
\hline \multirow[b]{2}{*}{ Period } & \multicolumn{4}{|c|}{ Linear Model } & \multicolumn{4}{|c|}{ GAM } \\
\hline & Intercept & Slope & $\mathrm{R}^{2}$ & $\overline{\mathrm{AIC}}$ & Intercept & EDF of $f(a a)$ & $\mathrm{R}^{2}$ & $\overline{\mathrm{AIC}}$ \\
\hline 1869-2009 & $0.36(0.29)$ & $0.005(0.014)$ & 0.00 & 424 & $0.47^{\mathrm{b}}(0.09)$ & $2.50^{\mathrm{c}}$ & 0.08 & $\overline{417}$ \\
\hline
\end{tabular}

${ }^{a}$ Standard errors for the intercept and slope of linear and the intercept for GAM models are in parentheses. The associated Akaike information criterion (AIC) and effective degree of freedom (EDF) for nonparametric smooth terms are listed for comparison.

${ }^{\mathrm{b}}$ Significant coefficients at the 0.001 level.

${ }^{\mathrm{c}}$ Significant coefficients at the 0.05 level. 

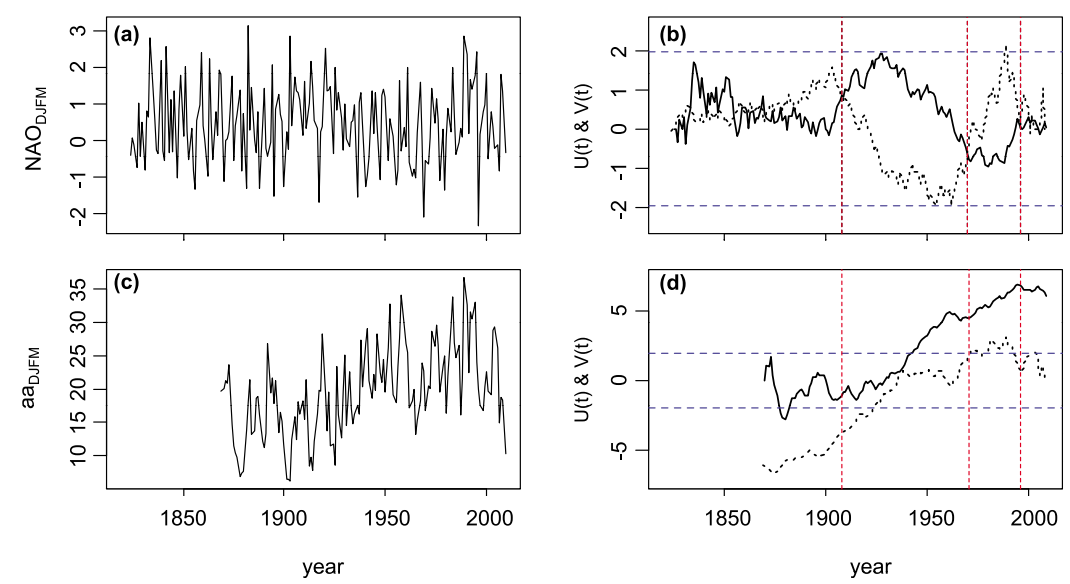

Figure 2. (a, c) Time evolution of NAO ${ }_{\text {DJFM }}$ and $a_{\text {DJFM }}$ for the period of 1800-2009. Note that $a a_{\text {DJFM }}$ started from 1869 while NAO $\mathrm{DJFM}_{\text {s. }}$ started from $1825 .(\mathrm{b}, \mathrm{d})$ The progressive $(U(t)$, solid) or retrograde $(V(t)$, dashed) scores of $\mathrm{NAO}_{\text {DJFM }}$ and $a a_{\text {DJFM }}$ calculated by using SMK test, where significant change points of the $\mathrm{NAO}_{\text {DJFM }}$ trend identified are shown as vertical red dashed lines and the confidence limits (i.e., \pm 1.96 ) are shown as horizontal blue dashed lines. No significant change of trend is detected for $a a_{\text {DJFM }}$.

[19] Figures $3 \mathrm{a}$ and $3 \mathrm{~b}$ show that the SMK-determined change points of the trend in the annual mean sunspot number Rz can be identified around 1812, 1844, 1903, and 1995 (vertical black dashed lines). The latter two change points of the Rz trend around 1903 and 1995 are remarkably close to those found in $\mathrm{NAO}_{\text {DJFM }}$ (shown as the vertical red lines taken from Figure 2b). Thus, our results show that change points of the trend in $\mathrm{Rz}$ occur just before those in the NAO while the SMK test found no long-term connection between $a a$ and the NAO (Figure 2d).

[20] Although both annual and winter mean $a a$ shows no significant change at multidecadal time scales, it does have a statistically significant statistical influence on the winter mean NAO at shorter time scales (i.e., interannual and decadal) according to the GAM (Figure 1a). Conversely, winter mean sunspot number $\mathrm{Rz}$ shows no relationship with the NAO on an interannual time scale but similar multidecadal trend change points as the NAO. To resolve this issue we applied the SMK test to a 5 year running correlation (corr $(a a, \mathrm{Rz}))$ between annual mean $\mathrm{Rz}$ and annual mean $a a$ (see Figure $3 \mathrm{c}$ ). It shows that $\operatorname{corr}(a a, \mathrm{Rz})$ fluctuates with time. It has been suggested that the long-term variation in $\mathrm{Rz}$ and $a a$ correlation may result from a $40 \pm$ 50 year quasiperiodicity of the time lag between solar activity and geomagnetic activity [Echer et al., 2004; Kishcha et al., 1999]. The rationale in the correlation between $\mathrm{Rz}$ and $a a$ used here is as a representation of the time varying response of the atmosphere to geomagnetic activity in the presence of solar UV conditioning as previously found by Lu et al. [2007]. Figure 3d shows that significant change points of the trend in $\operatorname{corr}(a a, \mathrm{Rz})$ are also found around 1903 and 1968 (Figures $3 \mathrm{c}$ and $3 \mathrm{~d}$ ).

[21] In addition, 1902 and 1957 are detected as significant change points of the trend of the December-March mean sunspot area in the Sun's north hemisphere (Figures $3 \mathrm{e}$ and 3f), and 1963 and 1993 are found as significant change points of the trend of north-south asymmetry of sunspot number As (Figures 3g and 3h). A common feature of these change points of the trends of the solar indices is that they all tend to precede the change points of $\mathrm{NAO}_{\mathrm{DJFM}}$ by between 0 and 12 years. These results suggest that changes of solar activity at a multidecadal scale might have an effect on the change of the winter NAO trend.

[22] In Figure 4, we investigate the nonstationary behavior of the $a a-\mathrm{NAO}$ relationship by subdividing the data sets into the four periods identified by the SMK tests. As a general rule, we chose to use those that represent the nearest change points to those detected in the NAO. However, as most of the change points in the solar indices identified by the SMK tests were around solar minimum except for the one around the 1960 s-1970s, we chose to use $1962 / 1963$ detected in solar asymmetry As rather than 1967/1968 detected in corr $(a a, \mathrm{Rz})$ as the separating years for the second and third periods. This is because 1963 was a solar minimum year while 1968 was closer to the solar maximum of SC 20. Nevertheless, this choice does not significantly change the conclusion in this paper.

[23] Figure 4 shows the GAMs fits for $\mathrm{NAO}_{\mathrm{DJFM}}$ and $a a_{\mathrm{DJFM}}$ over the subperiods of 1869-1902, 1903-1962, 1963-1995, and 1996-2009. The statistical measures of those models corresponding to Figure 4 are listed in Table 2 and compared with those from linear models (LMs). From Table 2 and Figure 4, it is evident from both linear and nonlinear models that the $a a-\mathrm{NAO}$ relation changes with time. For the two end periods of 1869-1902 and 1996-2009, no significant relationship between $a a_{\text {DJFM }}$ and $\mathrm{NAO}_{\text {DJFM }}$ can be established. For the period of 1903-1962, a significant negative linear realtion is found between $\mathrm{NAO}_{\mathrm{DJFM}}$ and $a a_{\text {DJFM }}$. Although this negative relationship is significant at the 0.01 level based on a LM, it may also be described as weakly nonlinear because slightly more of the variance in $\mathrm{NAO}_{\mathrm{DJFM}}$ can be explained by $a a_{\mathrm{DJFM}}$ when the GAM is used $\left(\mathrm{R}^{2}=0.17\right.$ for the GAM versus $\mathrm{R}^{2}=0.13$ for the $\left.\mathrm{LM}\right)$ and smaller AIC value. Thus, we can say that $17 \%$ of the variance in the $\mathrm{NAO}_{\text {DJFM }}$ can be explained by $a a_{\text {DJFM }}$ during this period. For the period of 1963-1995, a significant positive correlation is found between $\mathrm{NAO}_{\mathrm{DJFM}}$ and $a a_{\mathrm{DJFM}}$. The $a a$-NAO relationship for this subperiod is significant at the 0.001 level based on a linear model (LM). Again, such a relationship may also be described by a weak nonlinear 

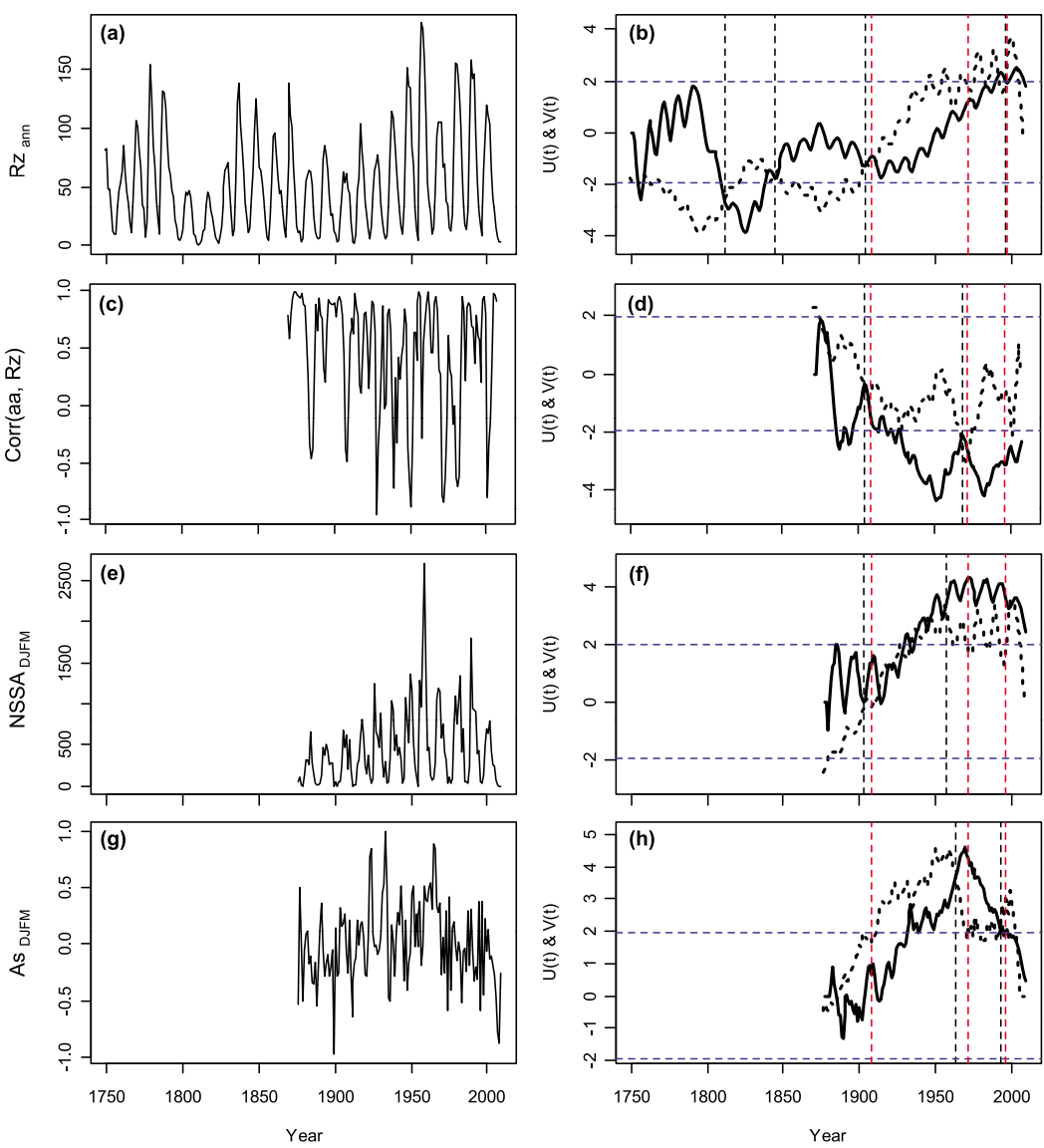

Figure 3. Same as Figure 2 but for annual mean sunspot number $\left(R z_{a n n}\right)(a, b)$ The 5 year running mean correlation between annual mean $a a$ and $\mathrm{Rz}_{\mathrm{ann}}(\operatorname{corr}(a a, \mathrm{Rz}))$, (c, d) December-March mean sunspot area in the Sun's northern hemisphere (NSSA DJFM), and (e, f) December-March mean sunspot number asymmetry $\left(A_{S}\right.$ DJFM $) .(\mathrm{g}, \mathrm{h})$ The years when the change points in the trends of $\mathrm{Rz}_{\mathrm{ann}}$, $\operatorname{corr}(a a, \mathrm{Rz}), \mathrm{NSSA}$ DJFM, and $A_{S}$ DJFM are shown as the vertical black dashed lines. For comparison, the years in which the changing points in the $\mathrm{NAO}_{\mathrm{DJFM}}$ trend are shown as vertical red dashed lines.

function because slightly more of the variance in $\mathrm{NAO}_{\mathrm{DJFM}}$ can be explained by $a a_{\text {DJFM }}$ by using GAM $\left(\mathrm{R}^{2}=0.50\right.$ for the GAM versus $\mathrm{R}^{2}=0.42$ for the $\mathrm{LM}$ ) and it produces a smaller AIC value. Thus we can say that $50 \%$ of the variance of the $\mathrm{NAO}_{\text {DJFM }}$ can be explained by $a a_{\text {DJFM }}$ during this period. These nonlinear concave shaped relationships remain significant at the 0.01 level, suggesting that the negative and positive relationship in the second and third periods (i.e., 1903-1962 and 1963-1995) contribute to form the overall concave-shaped $a a-\mathrm{NAO}$ relationship for the entire period of 1869-2009 (see Figure 1a). They further suggest that the $a a-\mathrm{NAO}$ relationship is not stationary over the last 140 years and that multidecadal variations of solar activity may be linked to the nonstationary behavior of the $a a-\mathrm{NAO}$ relationship.
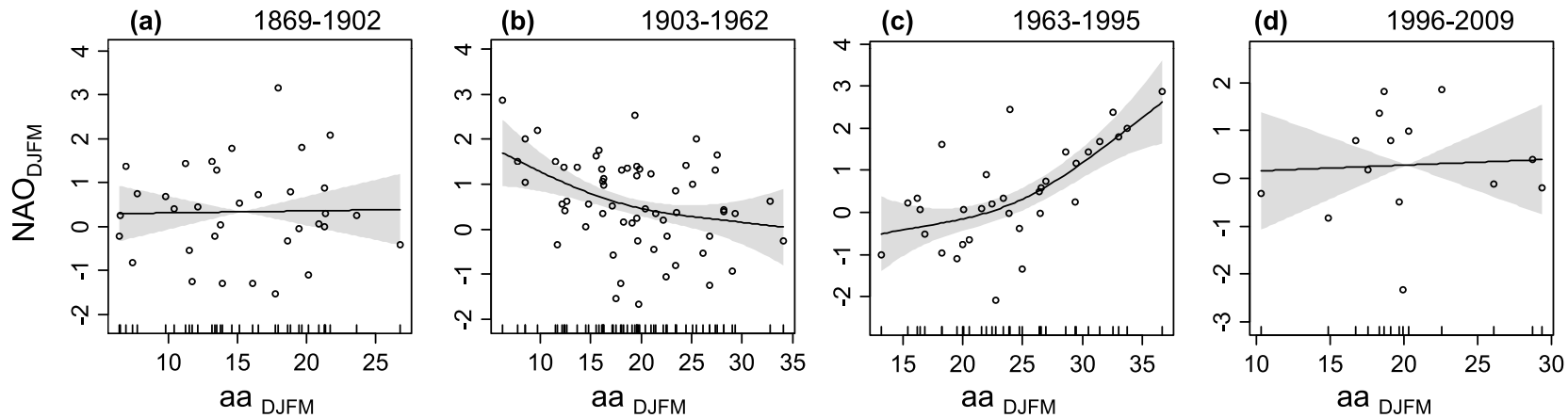

Figure 4. Same as Figure 1a but for the subperiods of (a) 1869-1902, (b) 1903-1962, (c) 1963-1995, and (d) 1996-2009. See Table 2 for detailed statistical estimates. 
Table 2. Same as Table 1 but for the Four Subperiods Identified by the SMK Test ${ }^{\mathrm{a}}$

\begin{tabular}{|c|c|c|c|c|c|c|c|c|}
\hline \multirow[b]{2}{*}{ Period } & \multicolumn{4}{|c|}{ Linear Model } & \multicolumn{4}{|c|}{ GAM } \\
\hline & Intercept & Slope & $\mathrm{R}^{2}$ & AIC & Intercept & EDF of $f(a a)$ & $\mathrm{R}^{2}$ & AIC \\
\hline 1869-1902 & $0.27(0.58)$ & $0.005(0.036)$ & 0.00 & 106 & $0.34(0.19)$ & 1 & 0.00 & 106 \\
\hline $1903-1962$ & $1.70^{\mathrm{b}}(0.40)$ & $-0.06^{\mathrm{c}}(0.02)$ & 0.13 & 168 & $0.60^{\mathrm{b}}(0.12)$ & $1.78^{\mathrm{c}}$ & 0.17 & 166 \\
\hline $1963-1995$ & $-2.68^{\mathrm{b}}(0.68)$ & $0.13^{\mathrm{b}}(0.03)$ & 0.42 & 92 & $0.43^{\mathrm{c}}(0.23)$ & $1.94^{\mathrm{b}}$ & 0.50 & 89 \\
\hline 1996-2009 & $0.33(1.30)$ & $0.01(0.06)$ & 0.00 & 48 & $0.28(0.31)$ & 1 & 0.00 & 48 \\
\hline
\end{tabular}

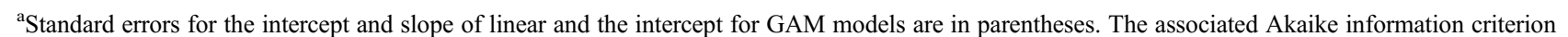
(AIC) and effective degree of freedom (EDF) for nonparametric smooth terms are listed for comparison.

${ }^{\mathrm{b}}$ Significant coefficients at the 0.001 level.

${ }^{\mathrm{c}}$ Significant coefficients at the 0.01 level.

\subsection{Effect of Recurrent Solar Wind at Different Phases of the SC}

[24] In this section, we investigate the $a a$-NAO relationship by separating the data according to odd- and evennumbered SCs and then focus on the even-numbered SCs, but separated by ascending and declining phases.

[25] Figure 5a shows how NAO $\mathrm{DJFM}_{\text {and }} a a_{\mathrm{DJFM}}$ is grouped into odd- (denoted by red circles) and even- (denoted by black circles) numbered cycles while Figure $5 \mathrm{~b}$ is the same as Figure 1a except that the data belong to odd- or evennumbered solar cycles are colored differently. Figure $5 \mathrm{c}$ shows that there is no statistical relationship between $\mathrm{NAO}_{\text {DJFM }}$ and $a a_{\text {DJFM }}$ for the odd-numbered SCs, while Figure $5 \mathrm{~d}$ sug- gests that there is significant $a a$-NAO relationship in evennumbered SCs. It is worth noting that the concave-shaped relationship is nearly identical to the original one without any data separation (see Figures $5 \mathrm{~b}$ and 1a). However, by excluding the data from odd-numbered solar cycles, the value of $\mathrm{R}^{2}$ increases from 0.08 to 0.17 (see the first two rows of Table 3), which confirms that a nonlinear relationship is again superior to the linear one. As a whole, results shown in Figure 5 suggest that the nonlinear $a a-\mathrm{NAO}$ is dominated by the data from the even-numbered SCs.

[26] Figure 6 examines even-numbered solar cycles in more detail. Figure 6a shows how we subdivided the data into ascending (red circles) and declining phases of even-
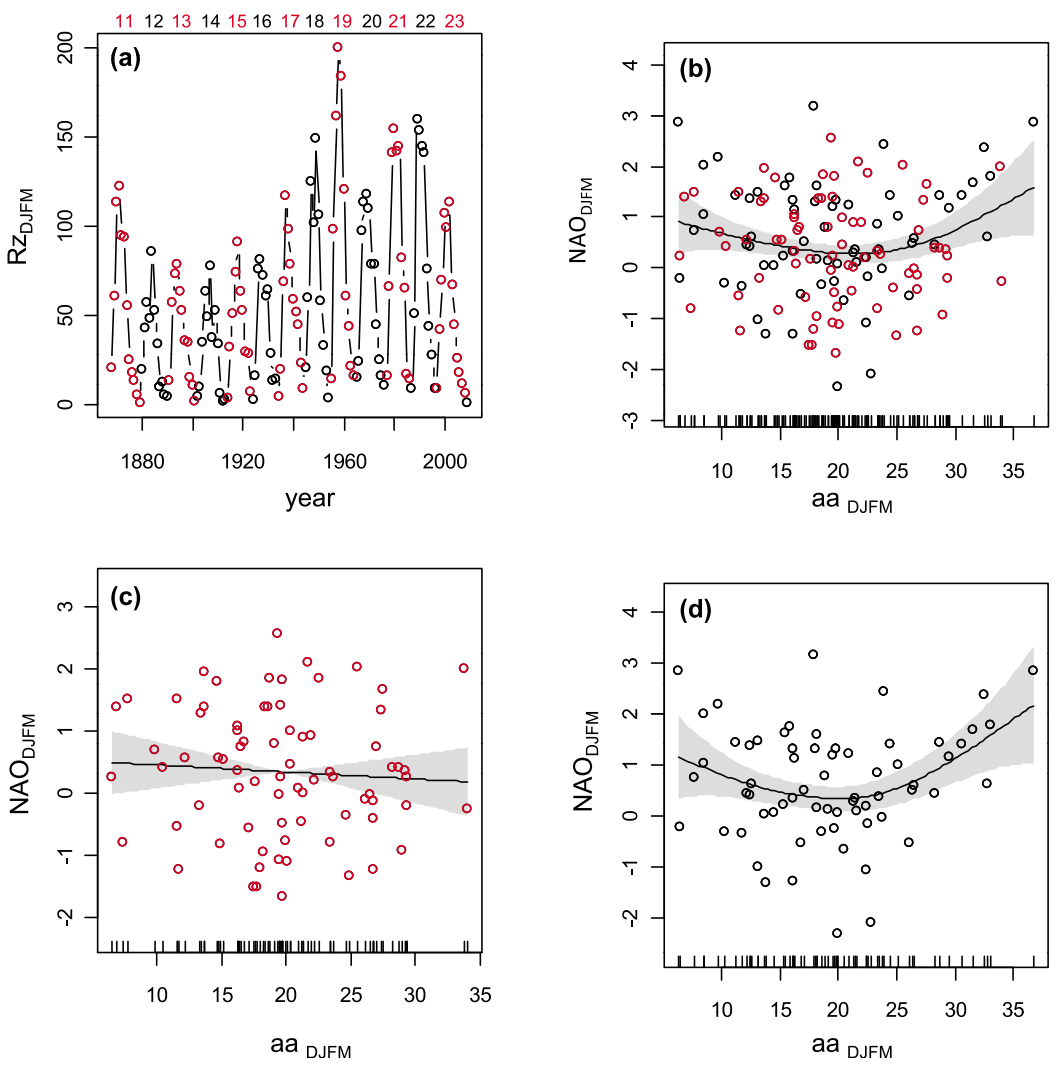

Figure 5. (a) Time series of December-March mean sunspot number $\left(\mathrm{Rz}_{\mathrm{DJFM}}\right)$ with the odd- and evennumbered SCs shown as red and black circles and the corresponding SC number at the top. (b) The GAM models built by using data for both odd and even-numbered SCs, (c) only using data from odd-numbered SCs, and (d) only using data from even-numbered SCs. See first two rows of Table 3 for detailed statistical estimates. 
Table 3. Same as Table 1 but Linear and GAM Models Were Built Based on the Data From Even (Odd)-Numbered SCs, the Declining (Ascending) Phases of Odd-Numbered SCs, and the Declining (Ascending) Phases of Even-Numbered SCs ${ }^{\mathrm{a}}$

\begin{tabular}{|c|c|c|c|c|c|c|c|c|}
\hline \multirow[b]{2}{*}{ Solar Cycle } & \multicolumn{4}{|c|}{ Linear Model } & \multicolumn{4}{|c|}{ GAM } \\
\hline & Intercept & Slope & $\mathrm{R}^{2}$ & AIC & Intercept & EDF of $f(a a)$ & $\mathrm{R}^{2}$ & AIC \\
\hline Odd-numbered SC & $0.56(0.40)$ & $-0.01(0.02)$ & 0.00 & 225 & $0.33^{\mathrm{b}}(0.11)$ & 1 & 0.00 & 225 \\
\hline Even-numbered SC & $0.27(0.41)$ & $0.02(0.02)$ & 0.01 & 206 & $0.64^{\mathrm{c}}(0.13)$ & $2.39^{\mathrm{d}}$ & 0.17 & 196 \\
\hline Ascending odd-numbered SC & $1.06(0.79)$ & $-0.04(0.04)$ & 0.04 & 96 & $0.24(0.19)$ & 1 & 0.04 & 96 \\
\hline Declining odd-numbered SC & $0.43(0.46)$ & $-0.001(0.02)$ & 0.00 & 132 & $0.40^{\mathrm{d}}(0.15)$ & 1 & 0.00 & 132 \\
\hline Ascending even-numbered SC & $0.97(0.71)$ & $-0.02(0.04)$ & 0.01 & 77 & $0.63^{\mathrm{d}}(0.22)$ & 4.59 & 0.40 & 74 \\
\hline Declining even-numbered SC & $-0.17(0.54)$ & $0.04(0.02)$ & 0.06 & 133 & $0.64^{\mathrm{c}}(0.14)$ & $2.43^{\mathrm{b}}$ & 0.34 & 121 \\
\hline
\end{tabular}

${ }^{\mathrm{a}}$ Standard errors for the intercept and slope of linear and the intercept for GAM models are in parentheses. The associated Akaike information criterion (AIC) and effective degree of freedom (EDF) for nonparametric smooth terms are listed for comparison.

${ }^{\mathrm{b}}$ Significant coefficients at the 0.01 level.

${ }^{\mathrm{c}}$ Significant coefficients at the 0.001 level.

${ }^{\mathrm{d}}$ Significant coefficients at the 0.05 level.

numbered SCs. Figure $6 \mathrm{~b}$ is the same as Figure $5 \mathrm{~d}$ but with data colored differently for ascending and declining phases. Figure $6 \mathrm{c}$ shows that there is an apparent nonlinear relationship between $\mathrm{NAO}_{\mathrm{DJFM}}$ and $a a_{\mathrm{DJFM}}$ for the ascending phase of even-numbered SCs. However, it is not statistically significant at the 0.05 level for the EDF (see the second last row of Table 3), so it may be coincidental. Figure 6d shows that the concave-shaped $a a-\mathrm{NAO}$ relationship becomes clearer and more robust statistically when only the data from the declining phase of even-numbered SCs are included. Its concave shape holds literally unchanged while it is signifi- cant at the 0.01 level compared with 0.05 when data from the ascending phases are included (see Figure $6 \mathrm{~b}$ and the second row of Table 3 ). It is worth noting that the value of $\mathrm{R}^{2}$ increases from 0.17 to 0.34 when the data from the ascending phases of even-numbered SCs were excluded (see Table 3 ). This represents $34 \%$ of the variances in the winter NAO under these conditions can be explained by the geomagnetic $a a$ index. A $\mathrm{R}^{2}=0.34$ is about 4 times of its original value $(0.08)$ where all data for the entire period of 1869-2009 were used (see Table 1). The progressive improvement of $\mathrm{R}^{2}$ and other statistical measures together
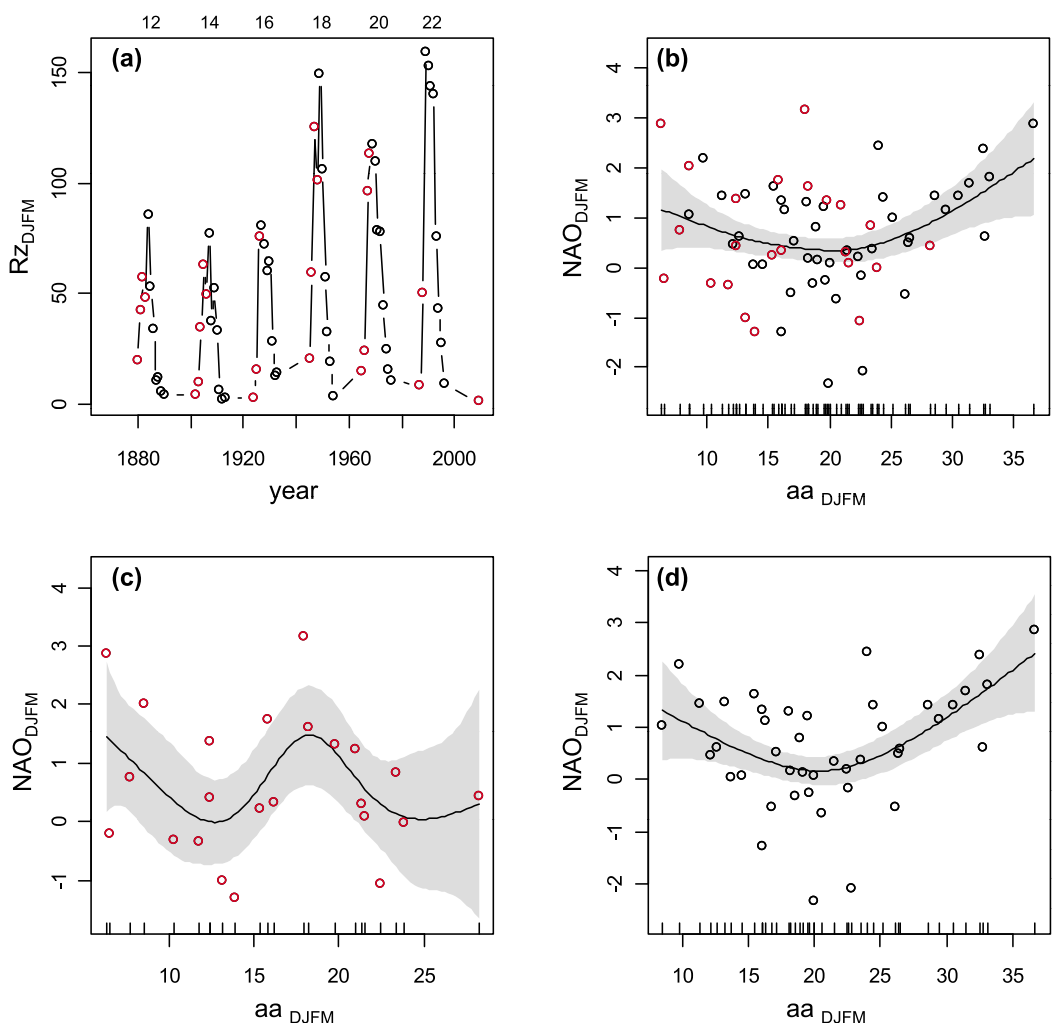

Figure 6. (a) Time series of $\mathrm{Rz}_{\mathrm{DJFM}}$ from all the even-numbered SCs with data in the ascending phase showing as red and declining phase as black circles and the corresponding even-numbered SC numbers at the top. (b) The GAM models built by using data both from ascending and declining phases of evennumbered SCs, (c) only using data from the ascending phase, and (d) only using data from the declining phase. See last four rows of Table 3 for detailed statistical estimates. 


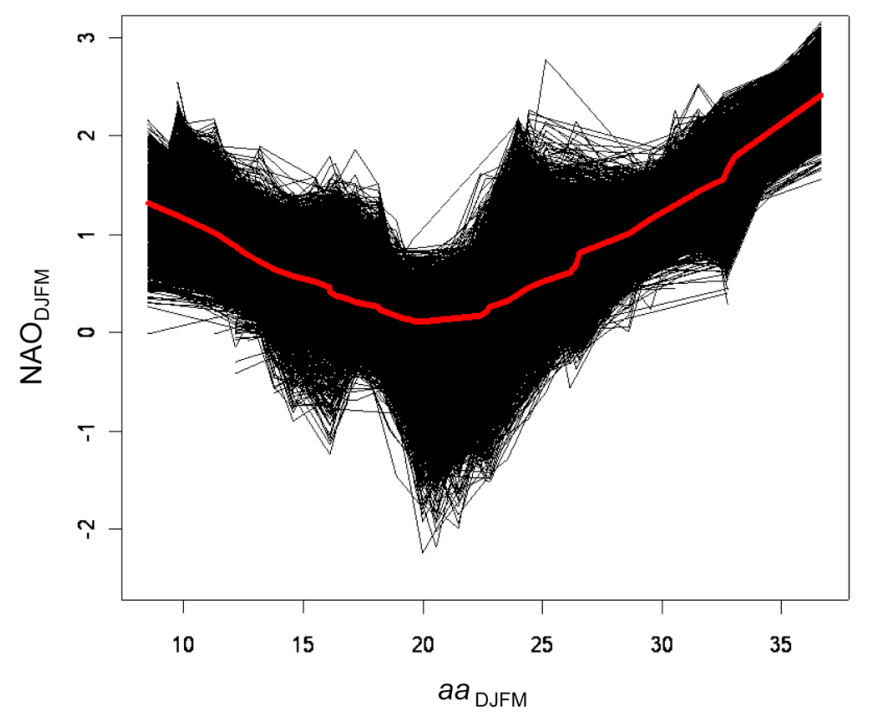

Figure 7. Bootstrap assessment of the winter $a a-\mathrm{NAO}$ relationship. The black lines represent the 10,000 GAMs built by sampling with replacement of the $a a$ and the NAO from the declining phase of even-numbered SCs. The mean fit of the 10,000 GAM bootstrapping models are marked as the red curve.

with little alteration in the concave-shaped relation strongly imply control of the data from the declining phase of evennumbered SCs.

[27] To further test the robustness of the winter $a a-\mathrm{NAO}$ relationship during the descending phase of even-numbered SCs, we have used a bootstrap technique to assess the uncertainty of the GAMs fitted to the $a a$-NAO relation. We have performed 10,000 bootstrap resamplings and built a GAM model for each sample. The resampling is done by sampling with replacement and only the $a a$ and the NAO data from the descending phase of even-numbered SCs are used. Figure 7 shows that the mean fit of the 10,000 GAM bootstrapping models and the associated uncertainty range are almost identical to those shown in Figure 6d. Thus, our bootstrap analysis further confirms that the concave relationship holds firmly during the descending phase of evennumbered solar cycles.

\section{Discussions}

[28] Many large-scale atmospheric variables including global temperature and the NAO exhibit multidecadal variations [Boudouridis et al., 2003; Delworth and Mann, 2000; Thompson et al., 2010]. However, little is known about what may have caused the multidecadal variability [Andronova and Schelsinger, 2000; Knight et al., 2006]. For the first time, we show that the geomagnetic effect on the winter NAO is nonstationary as well as nonlinear. Its nonstationary behavior is indicated by a decadal solar modulation of the shorter time scale relationship between geomagetic activity and the NAO. The multidecadel variation in the NAO may be partially linked to long-term solar variation for the last 180 years as the changes of the NAO trend seemed to synchronize or follow those in solar activity. It is unknown what has caused the multidecadal modulation. It might be attained by the solar UV effect on stratospheric ozone which, in turn, modulates the equator-to-pole temperature gradient in the stratosphere, which may modulate the atmospheric response to geomagnetic activity.

[29] Changing behavior of solar and/or geomagnetic activity has been reported before. Clilverd et al. [1998] showed a significant enhancement of geomagnetic activity since the beginning of 1900 s and suggested that the change was primarily caused by an increase in solar activity. Stamper et al. [1999] and Lockwood et al. [1999] suggested a doubling of Sun's coronal magnetic field during the 20th century.

[30] While on decadal to interannual time scales, a direct link between the NAO and the sunspot number cannot be found, there is however a weak but significant statistical connection between geomagnetic activity and the NAO over the period of 1869-2009. This indicates that on these shorter time scales, solar wind driven geomagnetic activity is more likely to affect the NAO than solar irradiance. This is consistent with the finding of Woollings et al. [2010b] who showed a solar effect in the eastern part of the North Atlantic that was much enhanced when open solar flux, rather than the F10.7 solar flux, was used to characterize the 11 year SC. Barriopedro et al. [2008] found that the 11 year SC modulates the preferred locations for high-pressure blocking occurrence over the Atlantic Ocean where blocking tends to be confined to either the western Atlantic under high solar conditions and over eastern Atlantic under low solar conditions. Together, these results suggest that the solar effect on the NAO is most likely of multiple sources operating on different time scales.

[31] The fact that a nonlinear $a a-\mathrm{NAO}$ relationship is dominated by the data from the declining phase of evennumbered SCs suggests high-speed recurrent solar wind streams may be the cause of the $a a-\mathrm{NAO}$ relationship. It was observed that the largest contribution of high-speed solar wind to auroral electron power tended to occur in the declining phases of the SCs [Cliver et al., 1996]. Cliver et al. [1996] found that the 22 year cycle in geomagnetic activity is characterized by higher activity during the second half of even-numbered SCs and the first half of odd number cycles. Those authors suggested that a 22 year variation of recurrent transient solar wind is due to cycle-to-cycle differences in the evolution of the global magnetic field of the Sun. They also suggested that 27 day recurrent wind streams were more prominent during the declining phase of even-numbered SCs, contributing to the higher geomagnetic activity observed at those times. These stronger recurrence patterns may be related to the more rapid expansion of polar coronal holes (faster movement of the coronal streamer belt to low latitudes) observed following the maxima of recent even-numbered cycles. Thus, the 22 year pattern of geomagnetic activity appears to be a reflection of the solar dynamo coupling of poloidal magnetic fields during the declining phase of one solar cycle to the toroidal fields at the maximum of the following cycle. This hypothesis has recently been supported by Shnirman et al. [2009] and by Georgieva and Kirov [2010].

[32] As shown in Figure 8, there was a significant increase in geomagnetic activity in the period from the beginning to the end of the 20th century, though a noticeable decrease of the daily $a a$ has been observed since the beginning of solar 


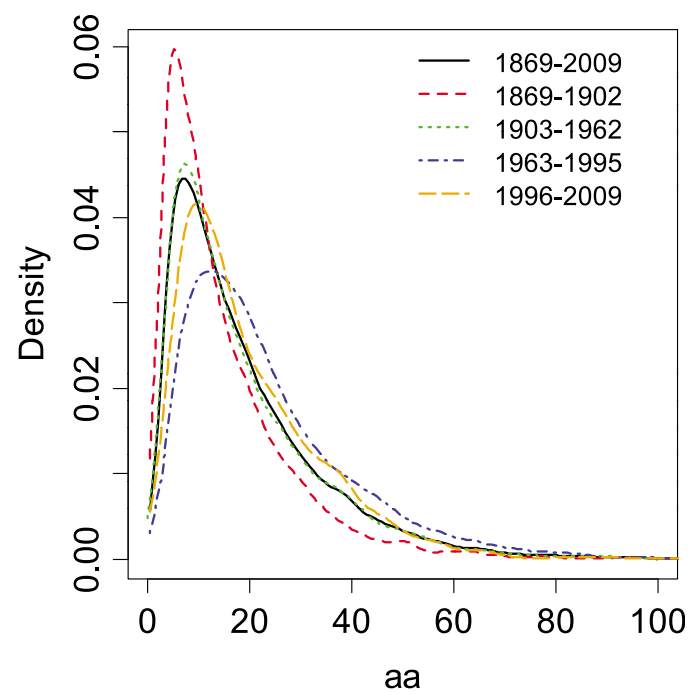

Figure 8. The density function of the daily mean $a a$ index for the entire period of 1868-2009 (black solid), and the four subperiods of 1868-1902 (red dashed), 1903-1962 (green dotted), 1963-1995 (blue dash-dotted), and 19952009 (orange long dashed). Note that only the probability density functions (PDFs) of the daily $a a$ index for the entire period (1868-2009) and subperiod 1903-1962 (green dotted) are not significantly different at the 0.05 level $(p$ value $=0.19)$ based on a Kolmogorov-Smirnoff test [Press et al., 1992]. The other PDFs are tested to be significantly different at the 0.001 level ( $p$ value $<0.001$ ). Moreover, according to the Wilcoxon-Mann-Whitney rank sum test [Iman, 1994], the mean values are also significantly different at the 0.001 level ( $p$ value $<0.001$ ) for all periods except that between the entire period 1868-2009 and the subperiod 1903-1962.

cycle 23. For the four subperiods studied here, 1963-1995 (blue dash-dotted line) stands out to be the most active period with the largest mean value and variance of the daily $a a$. The daily $a a$ occurred significantly more frequently for $a a=20-70 \mathrm{nT}$ during 1963-1995 than during the subperiod 1869-1902 (red dashed line). For the extreme values of $a a$ (e.g., daily $a a>80 \mathrm{nT}$ ), the differences between the four subperiods are small. As those extreme values of $a a$ are mostly associated with CME events while medium $a a$ values are associated with CIR events, Figure 8 further suggests that the enhancement of geomagnetic activity during 1963-1995 is likely due to an increase of high-speed solar wind during this time period.

[33] As shown in Figure 9, the $a a-\mathrm{NAO}$ relationship becomes stronger and most significant during the declining phases of even-numbered SC years for the subperiod 19631995 and significantly weaker for other subperiods. It is worth noting that, in Figures 9a and 9d, there are three outliers in 1969, 1995, and 1996; these were the years when the winter NAO values were extreme and the solar indices were undergoing a change in their trends. Excluding those years would significantly improve the $a a-\mathrm{NAO}$ relationship. For instance, for the subperiod 1963-1995, the $\mathrm{R}^{2}$ would increase from 0.59 to 0.92 and the $p$ value would decrease from 0.0008 to $1.67 \mathrm{e}-07$ if the two outliers (i.e., 1969 and
$1995)$ were excluded. It means that $59-92 \%$ of the winter NAO variance can be explained by $a a$ alone for the period of 1963-1995 and for those years in the declining phases of even-numbered SCs. As suggested by Figure 8, the subperiod 1963-1995 stands out to be the most activity period in terms of high-speed solar wind from CIR events. In addition, 27 day recurrent wind streams are more prominent during the decline of even-numbered solar cycles. Thus, the nonlinear behavior of the $a a$-NAO relationship may be due to a threshold response of the winter NAO to geomagnetic forcing. That is, such a detected geomagnetic effect on the NAO takes place only when there are strong and persistent high-speed solar wind streams emitted from the Sun. Below a certain threshold value of geomagnetic forcing, the effect becomes too weak to be detected statistically. This might be the reason that the significant positive correlation between $a a$ and the NAO only holds in 1970-1995 and collapsed before 1970 and after 1995, and why the relationship becomes clearer when the data from the declining phases of evennumbered SCs only are included.

[34] The fact that the $a a-\mathrm{NAO}$ relationship is dominated by the even-numbered SC may also be related to the RosenbergColeman (RC) effect [Rosenberg and Coleman, 1969]. The rotation axis of the Sun is tilted $7^{\circ}$ with respect to the ecliptic plane so that near the spring equinox in March the Earth is at maximum southern heliographic latitudes and near the equinox in September it is at maximum northern heliographic latitudes. Rosenberg and Coleman [1969] discovered that the dominant polarity of the IMF at times of most exposure to northern and southern latitudes is the same as the polarity of the corresponding pole on the Sun. Thus when the northern pole of the Sun is positive the IMF is away from the Sun above the heliographic equator. The Earth is above the Sun's equator around September and according to the $\mathrm{RC}$ effect will be dominated by IMF pointing away from the Sun. According to the RM rule [Russell and McPherron, 1973], this is a geoeffective orientation as it represents the most suitable IMF sector structure. Six months later the Earth will be at high southern latitudes where the IMF is toward the Sun. This situation is also geoeffective. Thus throughout an 11 year SC the ordinary behavior of the IMF is conducive to the production of variations in geomagnetic activity. However, it remains unclear why the $a a-\mathrm{NAO}$ relationship is particularly strong during the declining phases of the evennumbered solar cycles though it seems to link to persistent high-speed solar wind streams. More research is needed to clarify the underlying reasons for the dominant role of the declining phases of even-numbered SC in order to establish the observed $a a-\mathrm{NAO}$ relationship.

[35] It remains to be understood why the $a a-\mathrm{NAO}$ relationship switches from a negative effect for small to medium $a a$ and a positive one for medium to large $a a$. It might be due to the way that the geomagnetic forcing is coupled with Earth's atmosphere circulation and it may be producing a shift in atmospheric circulation. The presence of the solar UV conditioning as previously found by $L u$ et al. [2007] may also play a role in the solar modulation of the $a a-\mathrm{NAO}$ relationship at the multidecadal scale. To make the matter even more complicated, the connection between the 11 year $\mathrm{SC}$ and atmospheric variables can be further modulated by atmospheric internal variability such as the stratospheric 

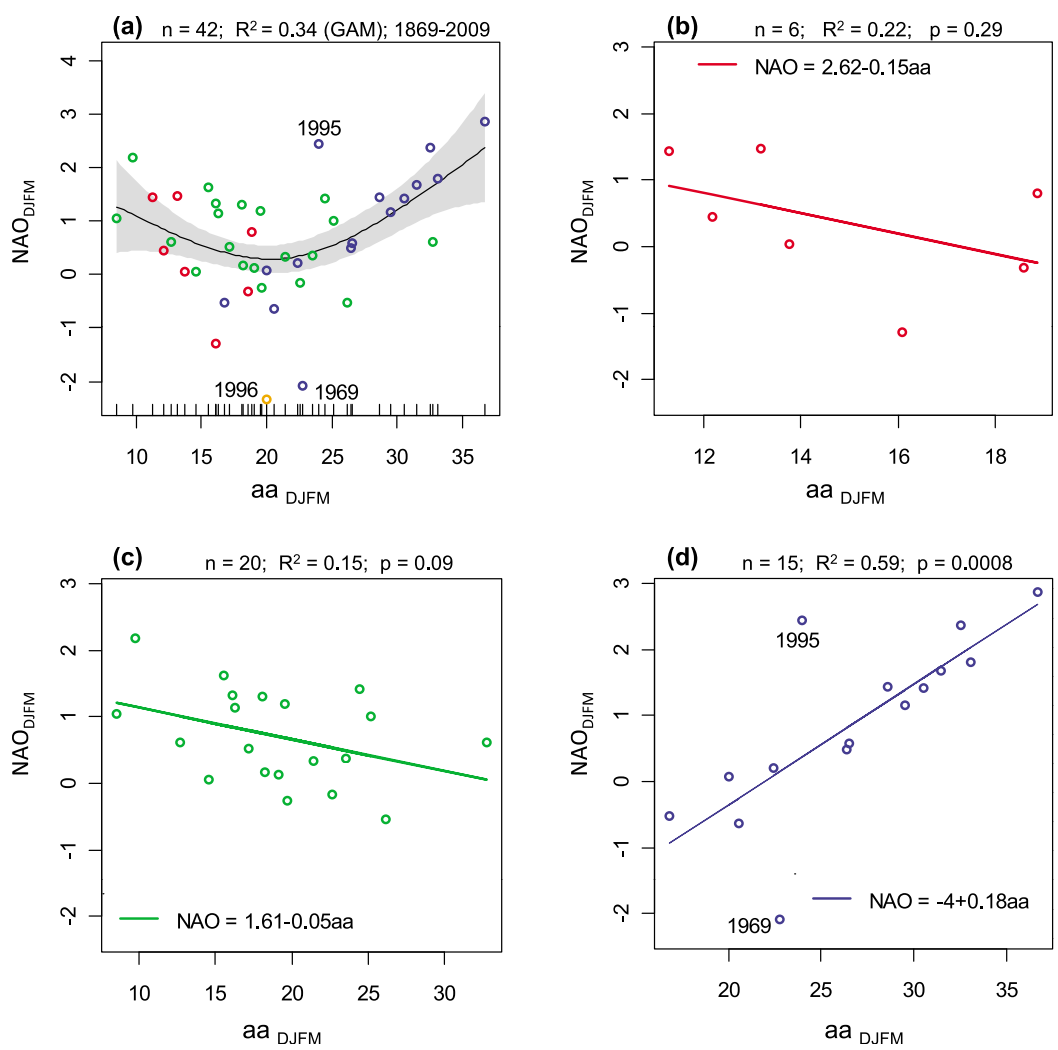

Figure 9. (a) Same as Figure 6d except that the data from the declining phases of even-numbered SCs within the subperiods of 1869-1902 (red circles), 1903-1962 (green circles), 1963-1995 (blue circles), and 1996-2009 (orange circles) are highlighted. Note that only 1 year (i.e., 1996) is in the declining phase of even-numbered SCs within the last subperiod of 1996-2009. Anomalous years (i.e., 1969, 1995, and 1996) were also labeled. Linear fitting of the $a a$-NAO relationship for the subperiods of (b) 1869-1902, (c) 1903-1962, and (d) 1963-1995 are shown, where the number of samples, estimated $R^{2}$ and $p$ value are given on the top. Anomalous years (i.e., 1969 and 1995) were also labeled in Figure 9d.

quasi-biennial oscillation [e.g., Labitzke, 1987; Lu et al., 2009].

\section{Conclusions}

[36] In this study, we have reexamined the statistical relationship between geomagnetic activity and the winter NAO. Our results indicate that a significant relationship can be established, which can be generally described by nonlinear concave shape with a negative relation for small to medium $a a$ and a positive relation for medium to large $a a$. We find that the $a a-\mathrm{NAO}$ relationship is also nonstationary such that it may be caused by a multidecadal modulation of solar activity. A multidecadal modulation of the winter time $a a-\mathrm{NAO}$ relationship by solar activity signifies that the Sun is more likely to affect Earth's climate by multiple means and the effects of solar and geomagnetic activity take place with different time scales. Thus, the Sun-climate connection is best viewed as a nonlinear and nonstationary process.

[37] The dominant role of the declining phases of evennumbered SCs in the $a a-\mathrm{NAO}$ relationship indicates that the 27 day recurrent solar wind streams may be responsible for the observed $a a-\mathrm{NAO}$ relationship. It is also possible that an increase of long-duration recurrent solar wind streams from high-latitude coronal holes during solar cycles 20 and 22 may partially account for the significant positive $a a-\mathrm{NAO}$ relationship during the latter third of the 20th century.

[38] More detailed studies are required to understand how the geomagnetic activity may affect the stratospheric and tropospheric circulation and wave activity, how those changes are communicated downward to influence the North Atlantic region, and to what extent the effect seen in our statistical analysis may be linked to known mechanisms such as energetic particle precipitation induced $\mathrm{NO}_{\mathrm{x}}$ [Randall et al., 2005; Solomon et al., 1982] and longer-term changes in stratosphere ozone [Haigh, 1994].

\section{Appendix A: Generalized Additive Model}

[39] The GAM is a nonparametric modeling technique that objectively estimates the functional relationship between the predictant and predictors in an additive model. In this study, we only consider a univariate model for detecting the relationship between a continuous predictant $Y_{t}$ (e.g., the NAO) and a predictor $X_{t}$ (e.g., the $a a$ index). As a result, the GAM fits a univariate regression model between the predictant $Y_{t}$ and predictor $X_{t}$,

$$
Y_{t}=\beta_{0}+f\left(X_{t}\right)+\varepsilon_{t}
$$


where $f$ is an unspecified smooth function to be estimated from observed data $\left\{\left(X_{t}, Y_{t}\right): t=1,2, \ldots, n\right\}, \beta_{0}$ is the constant (or intercept) and $\varepsilon_{t}$ the residual which follows a Gaussian distribution with zero mean and a constant variance. Note the error distribution may be relaxed to include not only a Gaussian, but all distributions from the exponential family (binomial, Poisson and Gamma). Here the error is assumed to follow a Gaussian distribution because the predictant (i.e., the NAO) is approximately Gaussian.

[40] There are several ways of defining the smooth function $f\left(X_{t}\right)$ and Hastie and Tibshirani [1990] give a useful overview of different types of smoothers. Here we use Wood's methods based on penalized regression smoothers [Wood, 2006]. Specifically, the smooth function $f\left(X_{t}\right)$ used in this study is of the form

$$
f\left(X_{t}\right)=\sum_{i=1}^{M} b_{i}\left(X_{t}\right) \beta_{i}
$$

where $b_{i}\left(X_{t}\right)$ are a set of cubic spline basis functions with $b_{1}\left(X_{t}\right)=X_{t}$ and $b_{i}\left(X_{t}\right)=\left|X_{t}-X_{i}^{*}\right|^{3}$ for $i=2, \ldots, M,\left\{X_{i}^{*}: j=\right.$ $2, \ldots, M\}$, and $\beta_{i}$ are the parameters to be estimated. Note that $X_{i}$ are a set of points in the range of $X_{t}$ known as the "knots" of the spline and $M$ is the basis dimension (i.e., the number of basis functions). Increasing $M$ allows $f\left(X_{t}\right)$ to track the data more closely while decreasing $M$ makes $f\left(X_{t}\right)$ to become more linear. For instance, substituting (A2) into (A1) with $M=1$ yields a linear model.

[41] Part of the GAM fitting process is to choose the appropriate degree of smoothness. Wood's method includes a penalty term in the model likelihood. This penalty term is controlled by a smoothing parameter $\lambda$ which determines the trade-off between the goodness of fit of the model and its smoothness. If $\lambda=0$, meaning there is no smoothing, it is equivalent to fitting the model using its original basis dimension. As $\lambda \rightarrow \infty$, there is a large amount of smoothing, which would result in a similar effect of choosing a lower basis dimension $M$. The smoothing parameter $\lambda$ is selected to minimize the generalized cross validation score $(\mathrm{GCV})$ [Craven and Wahba, 1979].

[42] Given the estimated smooth parameter $\lambda$, and the basis dimension $M$, the effective degree of freedom (EDF) for the smooth function $f\left(X_{t}\right)$ can be calculated. The EDF will be in the range of $[1, M]$ and is a measure of the complexity of the contribution of $X_{t}$ to $f\left(X_{t}\right)$ in the form of equation (A1). That is, $f\left(X_{t}\right)$ with $\mathrm{EDF}=1$ represents a straight line relationship and could be replaced by a liner term $\beta X_{t}$. As the EDF approaches $M$ the relationship is more complex. The smooth function $f\left(X_{t}\right)$ therefore gives the ability to examine the relationship between the predictant $Y_{t}$ and predictor $X_{t}$, whether they are linearly or nonlinear related. The "data-driven" estimated $f\left(X_{t}\right)$ is therefore most helpful to describe the unknown relationship between the predictant $Y_{t}$ and predictor $X_{t}$ when there is no prior knowledge [Underwood, 2009].

[43] Whether or not a fitted model is better or worse than another is determined by the Akaike Information Criterion (AIC) test statistic [Akaike, 1974]. AIC is a function of both the log likelihood function and the effective number of parameters being estimated [O'Brien and Rago, 1996]. For a $\mathrm{GAM}$ with $\mathrm{EDF}=k$, the $\mathrm{AIC}$ is calculated as

$$
\mathrm{AIC}=D+2 k \phi,
$$

where $D$ is the deviance and $\phi$ is the dispersion parameter (variance). The deviance estimated in the model, analogous to the residual sums of squares, is a measure of the fit of the model. A pseudo coefficient of determination, $\mathrm{R}^{2}$, is estimated as 1.0 minus the ratio of the deviance of the model to the deviance of the null model [Swartzman et al., 1992]. It is worth noting that the pseudo $\mathrm{R}^{2}$, is equivalent to the $\mathrm{R}^{2}$ for the models fitted with Gaussian error model. In this case, the deviance of the null model corresponds to the sum of total squares. Thus, the pseudo $\mathrm{R}^{2}$ is defined as the proportion of variation in $Y_{t}$ accounted for by the fitted model.

[44] Note that a linear model is nested within a GAM. The inference about the nonlinearity of the effect of a predictor is therefore accomplished by an analysis of deviance. Specifically, the nonlinearity of the effect of a given predictor (e.g., the $a a$ index) is tested by comparing the deviance of the $k$ degrees of freedom (df) smoothed model to the deviance of a $1 \mathrm{df}$ linear model [Hastie and Tibshirani, 1990]. The resulting nonparametric likelihood ratio test statistic is approximately distributed as a $\chi^{2}$ statistic with $k-1 \mathrm{dfs}$, which can be used to test the significance of the nonlinearity of $f\left(X_{t}\right)$ in terms of its EDF significantly bigger than 1 [Hastie and Tibshirani, 1990].

[45] A R package named $m g c v$ [Wood, 2006] provides easy access to the GAM technique described briefly above. Wood [2006] has also provided further detailed introduction, explanation and description of this method. The most recent development of this method is also available in the updated $m g c v$ package [Wood, 2008].

[46] All modeling results including AIC, EDF and $\mathrm{R}^{2}$, and the significance of the nonlinearity of $f\left(X_{t}\right)$ in this study were obtained by using the $m g c v$ package, and are given in Tables 1-3.

\section{Appendix B: Sequential Mann-Kendall Test}

[47] The sequential Mann-Kendall test is a statistical technique to determine potential trend change in a time series. Following Gerstengarbe and Werner [1999], let a time series $\mathbf{X}=\left\{X_{1}, \ldots, X_{n}\right\}$ be separated into $n-1$ subseries (i.e., the first subseries includes the sample values $X_{1}$, $X_{2}$, the second include the values $X_{1}, X_{2}, X_{3}$ etc). The $n-1$ Mann-Kendall test statistic variables [Mann, 1945] determined by these subseries are given as

$$
W_{t}=\sum_{i=1}^{t} R_{i}
$$

where $R_{i}$ is the rank of the $t$-th subseries $\left\{X_{1}, X_{2}, \cdots, X_{t+1}\right\}$, i.e., the number of the elements $X_{i}(i>j)$ such that $X_{i}>X_{j}$ with $i=2, \ldots, t$ and $j=1, \ldots, i-1$. Consequently, for each of the $n-1$ subseries, the corresponding progressive row $U(t)$ is defined as

$$
U(t)=\frac{W_{t}-E\left(W_{t}\right)}{\sqrt{\operatorname{Var}\left(W_{t}\right)}},
$$


where $E\left(W_{t}\right)$ is the expected value of the respective subseries with

$$
E\left(W_{t}\right)=\frac{l_{t}\left(l_{t}-1\right)}{4}
$$

and $\operatorname{Var}\left(W_{t}\right)$ is the respective variance given by

$$
\operatorname{Var}\left(W_{t}\right)=\frac{l_{t}\left(l_{t}-1\right)\left(2 l_{t}-5\right)}{72}
$$

For $l_{t} \rightarrow \infty\left(l_{t}=\right.$ the length of the subseries $), W_{t}$ is approximately Gaussian [see Gerstengarbe and Werner, 1999] and the normalized $U(t)$ is assumed to be a standard Gaussian distribution. Similarly, the test statistic variable $V(t)$ can be defined and calculated by using the corresponding rank series for the so-called retrograde rows for the resorted sample $\left\{X_{t+1}, X_{t}, \cdots, X_{1}\right\}$ for $t=1, \ldots, n-1$.

[48] After the test statistic variables $U(t)$ and $V(t)$ are calculated for all progressive and retrograde series, one obtains $n-1$ corresponding values of so-called the progressive and retrograde rows, respectively. Points where the two rows of $U(t)$ and $V(t)$ cross are considered as potential trend turning points within the time series. When either the progressive $U(t)$ or retrograde $V(t)$ row exceeds certain confidence limits before and after the crossing point, the null hypothesis (the sampled time series has no change points) must be rejected, and this trend turning point is considered significant at the corresponding level, i.e., 1.96 for $95 \%$ significant level.

[49] Acknowledgments. Y.L. and B.B. were supported by the Indian Ocean Climate Initiative. H.L., M.J.J., and M.A.C. were supported by the UK Natural Environment Research Council (NERC). We thank two anonymous reviewers for their constructive comments.

\section{References}

Akaike, H. (1974), A new look at the statistical model identification, IEEE Trans. Autom. Control, 19(6), 716-723, doi:10.1109/TAC.1974.1100705. Andronova, N. G., and M. E. Schelsinger (2000), Causes of global temperature changes during the 19th and 20th centuries, Geophys. Res. Lett., 27, 2137-2140, doi:10.1029/2000GL006109.

Arnold, N. F., and T. R. Robinson (2001), Solar magnetic flux influences on the dynamics of the winter middle atmosphere, Geophys. Res. Lett., 28, 2381-2384, doi:10.1029/2000GL012825.

Badalyan, O. G., and V. N. Obridko (2011), North-south asymmetry of the sunspot indices and its quasi-biennial oscillations, New Astron., 16, 357-365, doi:10.1016/j.newast.2011.01.005.

Barriopedro, D., R. Garćia-Herrera, and R. Huth (2008), Solar modulation of Northern Hemisphere winter blocking, J. Geophys. Res., 113, D14118, doi:10.1029/2008JD009789.

Boberg, F., and H. Lundstedt (2002), Solar wind variations related to fluctuations of the North Atlantic Oscillation, Geophys. Res. Lett., 29(15), 1718, doi:10.1029/2002GL014903.

Boberg, F., and H. Lundstedt (2003), Solar wind electric field modulation of the NAO: A correlation analysis in the lower atmosphere, Geophys. Res. Lett., 30(15), 1825, doi:10.1029/2003GL017360.

Bochníček, J., and P. Hejda (2005), The winter NAO pattern changes in association with solar and geomagnetic activity, J. Atmos. Sol. Terr. Phys., 67, 17-32, doi:10.1016/j.jastp.2004.07.014.

Boudouridis, A., E. Zesta, L. R. Lyons, P. C. Anderson, and D. Lummerzheim (2003), Effect of soalr wind pressure pulses on the size and strength of the auroral oval, J. Geophys. Res., 108(A4), 8012, doi:10.1029/ 2002JA009373.

Bucha, V., and V. Bucha Jr. (1998), Geomagnetic forcing of changes in climate and in the atmospheric circulation, J. Atmos. Sol. Terr. Phys., 60, 145-169, doi:10.1016/S1364-6826(97)00119-3.
Bumba, V., and L. Hejna (1991), A new index of recurrence for long-lasting enhanced geomagnetic activity, Bull. Astron. Inst. Czech., 42(2), 85-90.

Clilverd, M. A., T. D. G. Clark, E. Clarke, and H. Rishbeth (1998), Increased magnetic storm activity from 1868 to 1995, J. Atmos. Sol. Terr. Phys., 60, 1047-1056, doi:10.1016/S1364-6826(98)00049-2.

Cliver, E. W., V. Boriakoff, and K. H. Bounar (1996), The 22-year cycle of geomagnetic and solar wind activity, J. Geophys. Res., 101, 27,09127,109, doi:10.1029/96JA02037.

Corti, S., F. Molteni, and T. N. Palmer (1999), Signature of recent climate change in frequencies of natural atmospheric circulation regimes, Nature, 398, 799-802, doi:10.1038/19745.

Cox, M. E., A. Moss, and G. K. Smyth (2005), Water quality condition and trend in North Queensland waterways, Mar. Pollut. Bull., 51(1-4), 89-98, doi:10.1016/j.marpolbul.2004.10.039.

Craven, P., and G. Wahba (1979), Smoothing noisy data with spline functions, Numer. Math., 31(4), 377-403, doi:10.1007/BF01404567.

Delworth, T. L., and M. E. Mann (2000), Observed and simulated multidecadal variability in the Northern Hemisphere, Clim. Dyn., 16, 661-676, doi: $10.1007 / \mathrm{s} 003820000075$.

Echer, E., W. D. Gonzalez, A. L. C. Gonzalez, A. Prestes, L. E. A. Vieira, A. Dal Lago, F. L. Guarnieri, and N. J. Schuch (2004), Long-term correlation between solar and geomagnetic activity, J. Atmos. Sol. Terr. Phys., 66, 1019-1025, doi:10.1016/j.jastp.2004.03.011.

Emery, B. A., I. G. Richardson, D. S. Evans, and F. J. Rich (2009), Solar wind structure sources and periodicities of auroral electron power over three solar cycles, J. Atmos. Sol. Terr. Phys., 71, 1157-1175, doi:10.1016/j.jastp.2008.08.005.

Enfield, D. B., A. M. Mestas-Nuñez, and P. J. Trimble (2001), The Atlantic Multidecadal Oscillation and its relation to rainfall and river flows in the continental US, Geophys. Res. Lett., 28, 2077-2080, doi:10.1029/ 2000GL012745.

Feldstein, S. B. (2002), The recent trend and variance increase of the annular mode, J. Clim., 15, 88-94, doi:10.1175/1520-0442(2002)015<0088: TRTAVI $>2.0 . \mathrm{CO} ; 2$.

Georgieva, K., and B. Kirov (2010), Solar dynamo and geomagnetic activity, J. Atmos. Sol. Terr. Phys., 73, 207-222, doi:10.1016/j.jastp.2010.03.003. Gerstengarbe, F. W., and P. C. Werner (1999), Estimation of the beginning and end of recurrent events within a climate regime, Clim. Res., 11, 97-107, doi:10.3354/cr011097.

Gillett, N. P., F. W. Zwiers, A. J. Weaver, and P. A. Stott (2003), Detection of human influence on sea-level pressure, Nature, 422, 292-294, doi:10.1038/nature01487.

Gong, G., D. Entekhabi, and J. Cohen (2002), A large-ensemble model study of the wintertime AO-NAO and the role of interannual snow perturbations, J. Clim., 15, 3488-3499, doi:10.1175/1520-0442(2002) 015<3488:ALEMSO>2.0.CO;2.

Gong, G., D. Entekhabi, and J. Cohen (2003), Modeled Northern Hemisphere winter climate response to realistic Siberian snow anomalies, J. Clim., 16, 3917-3931, doi:10.1175/1520-0442(2003)016<3917: MNHWCR $>2.0 . \mathrm{CO} ; 2$.

Gray, S. T., L. J. Graumlich, J. L. Betancourt, and G. T. Pederson (2004), A tree-ring based reconstruction of the Atlantic Multidecadal Oscillation since 1567 A.D., Geophys. Res. Lett., 31, L12205, doi:10.1029/ 2004GL019932.

Gu, C. (1992), Cross-validating non-Gaussian data, J. Comput. Graphical Stat., 1, 169-179, doi:10.2307/1390840.

Haigh, J. D. (1994), The role of stratospheric ozone in modulating the solar radiative forcing of climate, Nature, 370, 544-546, doi:10.1038/ $370544 \mathrm{a} 0$.

Hall, A., and M. Visbeck (2002), Synchronous variability in the Southern Hemisphere atmosphere, sea ice, and ocean resulting from the annular mode, J. Clim., 15, 3043-3057, doi:10.1175/1520-0442(2002) 015<3043:SVITSH $>2.0 . \mathrm{CO} ; 2$.

Hapgood, M. A. (1993), A double solar cycle in the 27-day recurrence of geomagnetic activity, Ann. Geophys., 11, 248-253.

Harrison, R. G., and D. B. Stephenson (2006), Empirical evidence for a nonlinear effect of galactic cosmic rays on clouds, Proc. R. Soc. A, 462, 1221-1233, doi:10.1098/rspa.2005.1628.

Hastie, T., and R. Tibshirani (1990), Generalized Additive Models, Monogr. Stat. Appl. Probab. Ser., vol. 43, Chapman and Hall, London.

He, S., S. Mazumdar, and V. C. Arena (2006), A comparative study of the use of GAM and GLM in air pollution research, Environmetrics, 17, 81-93, doi:10.1002/env.751.

Hoerling, M. P., J. W. Hurrell, and T. Y. Xu (2001), Tropical origins for recent North Atlantic climate change, Science, 292, 90-92, doi:10.1126/ science. 1058582 .

Hurrell, J. W. (1995), Decadal trends in the North Atlantic Oscillation: Regional temperatures and precipitation, Science, 269, 676-679, doi:10.1126/science.269.5224.676. 
Hurrell, J. W., and C. Deser (2010), North Atlantic climate variability: The role of the North Atlantic Oscillation, J. Mar. Syst., 79, 231-244, doi:10.1016/j.jmarsys.2009.11.002.

Hurrell, J. W., Y. Kushnir, G. Ottersen, and M. E. Visbeck (Eds.) (2003), The North Atlantic Oscillation: Climate Significance and Environmental Impact, Geophys. Monogr. Ser., vol. 134, 279 pp., AGU, Washington, D. C.

Iman, R. L. (1994), A Data-Based Approach to Statistics, 348 pp., Duxbury, Pacific Grove, Calif.

Jones, P. D., T. Jónsson, and D. Wheeler (1997), Extension to the North Atlantic Oscillation using early instrumental pressure observations from Gibraltar and south-west Iceland, Int. J. Climatol., 17, 1433-1450, doi:10.1002/(SICI) 1097-0088(19971115)17:13<1433::AID-JOC203>3.0.CO;2-P.

Kishcha, P. V., I. V. Dmitrieva, and V. N. Obridko (1999), Long-term variations of the solar-geomagnetic correlation, total solar irradiance, and Northern Hemispheric temperature (1868-1997), J. Atmos. Sol. Terr. Phys., 61, 799-808, doi:10.1016/S1364-6826(99)00035-8.

Knight, J. R., C. K. Folland, and A. A. Scaife (2006), Climate impacts of the Atlantic Multidecadal Oscillation, Geophys. Res. Lett., 33, L17706, doi:10.1029/2006GL026242.

Kniveton, D. R., B. A. Tinsley, G. B. Burns, E. A. Bering, and O. A. Troshichev (2008), Variations in global cloud cover and the fair-weather vertical electric field, J. Atmos. Sol. Terr. Phys., 70, 1633-1642, doi:10.1016/j.jastp.2008.07.001.

Kodera, K. (2002), Solar cycle modulation of the North Atlantic Oscillation: Implication in the spatial structure of the NAO, Geophys. Res. Lett. 29(8), 1218, doi:10.1029/2001GL014557.

Kodera, K. (2003), Solar influence on the spatial structure of the NAO during the winter 1900-1999, Geophys. Res. Lett., 30(4), 1175, doi:10.1029/ 2002GL016584.

Labitzke, K. (1987), Sunspots, the QBO, and the stratospheric temperature in the north polar-region, Geophys. Res. Lett., 14, 535-537, doi:10.1029/ GL014i005p00535.

Laken, B., A. Wolfendale, and D. Kniveton (2009), Cosmic ray decreases and changes in the liquid water cloud fraction over the oceans, Geophys. Res. Lett., 36, L23803, doi:10.1029/2009GL040961.

Li, K. J., J. X. Wang, S. Y. Xiong, H. F. Liang, H. S. Yun, and X. M. Gu (2002), Regularity of the north-south asymmetry of solar activity, Astron. Astrophys., 383(2), 648-652, doi:10.1051/0004-6361:20011799.

Liu, Y., C. J. Paciorek, and P. Koutrakis (2009), Estimating regional spatial and temporal variability of PM2.5 concentrations using satellite data, meteorology, and land use information, Environ. Health Perspect., 117(6), 886-892.

Lockwood, M., R. Stamper, and M. N. Wild (1999), A doubling of the Sun's coronal magnetic field during the last 100 years, Nature, 399, 437-439, doi:10.1038/20867.

Lu, H., M. J. Jarvis, H.-F. Graf, P. C. Young, and R. B. Horne (2007), Atmospheric temperature responses to solar irradiance and geomagnetic activity, J. Geophys. Res., 112, D11109, doi:10.1029/2006JD007864.

Lu, H., M. A. Clilverd, A. Seppälä, and L. L. Hood (2008a), Geomagnetic perturbations on stratospheric circulation in late winter and spring, J. Geophys. Res., 113, D16106, doi:10.1029/2007JD008915.

Lu, H., M. J. Jarvis, and R. E. Hibbins (2008b), Possible solar wind effect on the Northern Annular Mode and Northern Hemispheric circulation during winter and spring, J. Geophys. Res., 113, D23104, doi:10.1029/ 2008JD010848

Lu, H., L. J. Gray, M. P. Baldwin, and M. J. Jarvis (2009), Life cycle of the QBO-modulated 11-year solar cycle signals in the Northern Hemispheric winter, Q. J. R. Meteorol. Soc., 135, 1030-1043, doi:10.1002/qj.419.

Mann, H. B. (1945), Nonparametric test against trend, Econometrica, 13, $245-259$.

Mayaud, P.-N. (1972), The $a a$ indices: A 100-year series characterizing the magnetic activity, J. Geophys. Res., 77, 6870-6874, doi:10.1029/ JA077i034p06870.

McLeod, S. R., and A. R. Pople (2010), Modelling the distribution and relative abundance of feral camels in the Northern Territory using count data, Rangeland J., 32(1), 21-32, doi:10.1071/RJ09057.

Mehta, V. M., M. J. Suarez, J. V. Manganello, and T. L. Delworth (2000), Oceanic influence on the North Atlantic Oscillation and associated Northern Hemisphere climate variations: 1959-1993, Geophys. Res. Lett., 27, 121-124, doi:10.1029/1999GL002381.

Mestre, O., and S. Hallegatte (2009), Predictors of tropical cyclone numbers and extreme hurricane intensities over the North Atlantic using generalized additive and linear models, J. Clim., 22, 633-648, doi:10.1175/ 2008JCLI2318.1.

Mikolajczyk, R. T., A. E. Maxwell, W. El Ansari, C. Stock, J. Petkeviciene, and F. Guillen-Grima (2010), Relationship between perceived body weight and body mass index based on self-reported height and weight among university students: A cross-sectional study in seven European countries, BMC Public Health, 10, 1-40, doi:10.1186/1471-2458-10-40.

Morton, R., and B. L. Henderson (2008), Estimation of nonlinear trends in water quality: An improved approach using generalized additive models, Water Resour. Res., 44, W07420, doi:10.1029/2007WR006191.

O'Brien, L., and P. Rago (1996), An application of the generalized additive model to groundfish Survey Data with Atlantic cod off the northeast coast of the United States as an example, NAFO Sci. Counc. Stud., 28, 79-95.

Ogi, M., K. Yamazaki, and Y. Tachibana (2003), Solar cycle modulation of the seasonal linkage of the North Altantic Oscillation (NAO), Geophys. Res. Lett., 30(22), 2170, doi:10.1029/2003GL018545.

Osborn, T. J. (2004), Simulating the winter North Atlantic Oscillation: The roles of internal variability and greenhouse gas forcing, Clim. Dyn., 22, 605-623.

Palamara, D. R., and E. A. Bryant (2004), Geomagnetic activity forcing of the Northern Annular Mode via the stratosphere, Ann. Geophys., 22 , 725-731, doi:10.5194/angeo-22-725-2004.

Paluš, M., and D. Novotná (2007), Common oscillatory modes in geomagnetic activity, NAO index and surface air temperature records, J. Atmos. Sol. Terr. Phys. 69, 2405-2415, doi:10.1016/j.jastp.2007.05.009.

Paluš, M., and D. Novotná (2009), Phase-coherent oscillatory modes in solar and geomagnetic activity and climate variability, J. Atmos. Sol. Terr. Phys., 71, 923-930, doi:10.1016/j.jastp.2009.03.012.

Pierce, J. R., and P. J. Adams (2009), Can cosmic rays affect cloud condensation nuclei by altering new particle formation rates?, Geophys. Res. Lett., 36, L09820, doi:10.1029/2009GL037946.

Press, W. H., B. P. Flannery, S. A. Teukolsky, and W. T. Vetterling (1992), Numerical Recipes in C: The Art of Scientific Computing, 2nd ed., 848 pp., Cambridge Univ. Press, New York.

Pudovkin, M. I., and S. V. Veretenenko (1995), Cloudiness decreases associated with Forbush-decreases of galactic cosmic rays, J. Atmos. Sol. Terr. Phys., 57, 1349-1355, doi:10.1016/0021-9169(94)00109-2.

Pudovkin, M. I., and S. V. Veretenenko (1996), Variations of the cosmic rays as one of the possible links between the solar activity and the lower atmosphere, Adv. Space Res., 17, 161-164, doi:10.1016/02731177(95)00745-Z.

Randall, C. E., et al. (2005), Stratospheric effects of energetic particle precipitation in 2003-2004, Geophys. Res. Lett., 32, L05802, doi:10.1029/ 2004GL022003

Randall, C. E., V. L. Harvey, C. S. Singleton, P. F. Bernath, C. D. Boone, and J. U. Kozyra (2006), Enhanced $\mathrm{NO}_{\mathrm{x}}$ in 2006 linked to strong upper stratospheric Arctic vortex, Geophys. Res. Lett., 33, L18811, doi:10.1029/2006GL027160.

Randall, C. E., V. L. Harvey, C. S. Singleton, S. M. Bailey, P. F. Bernath, M. Codrescu, H. Nakajima, and J. M. Russell III (2007), Energetic particle precipitation effects on the Southern Hemisphere stratosphere in 1992-2005, J. Geophys. Res., 112, D08308, doi:10.1029/2006JD007696.

Rangarajan, G. K. (1991), Variations in the strength of recurrent geomagnetic activity in solar cycles 11 to 21, Proc. Indian Acad. Sci. Earth Planet. Sci., 100, 49-54.

Rodwell, M. J., D. P. Rowell, and C. K. Folland (1999), Oceanic forcing of the wintertime North Atlantic Oscillation and European climate, Nature, 398, 320-323, doi: $10.1038 / 18648$.

Rosenberg, R., and P. J. Coleman Jr. (1969), Heliographic latitude dependence of the dominant polarity of the interplanetary magnetic field, J. Geophys. Res., 74, 5611-5622, doi:10.1029/JA074i024p05611.

Russell, C. T., and R. L. McPherron (1973), Semiannual variation of geomagnetic activity, J. Geophys. Res., 78, 92-108, doi:10.1029/ JA078i001p00092.

Saenger, C., A. L. Cohen, D. W. Oppo, R. B. Halley, and J. E. Carilli (2009), Surface-temperature trends and variability in the low-latitude North Atlantic since 1552, Nat. Geosci., 2, 492-495, doi:10.1038/ ngeo552.

Sargent, H. H., III (1985), Recurrent geomagnetic activity: Evidence for long-lived stability in solar wind structure, J. Geophys. Res., 90, 1425-1428, doi:10.1029/JA090iA02p01425.

Sargent, H. H., III (1986), The 27-day recurrence index, in Solar Wind Magnetosphere Coupling, edited by Y. Kamide and J. A. Slavin, pp. 143-148, Terra Sci., Tokyo.

Seppälä, A., C. E. Randall, M. A. Clilverd, E. Rozanov, and C. J. Rodger (2009), Geomagnetic activity and polar surface air temperature variability, J. Geophys. Res., 114, A10312, doi:10.1029/2008JA014029.

Shaviv, N. J. (2005), On climate response to changes in the cosmic ray flux and radiative budget, J. Geophys. Res., 110, A08105, doi:10.1029/ 2004JA010866.

Shindell, D. T., R. L. Miller, G. A. Schmidt, and L. Pandolfo (1999), Simulation of recent northern winter climate trends by greenhouse-gas forcing, Nature, 399, 452-455, doi:10.1038/20905. 
Shnirman, M. G., J.-L. L. Mouël, and E. M. Blanter (2009), The 27-day and 22-year cycles in solar and geomagnetic activity, Solar. Phys., 258, 167-179, doi:10.1007/s11207-009-9395-9.

Sneyers, R. (1990), On the Statistical Analysis of Series of Observations, 143 pp., World Meteorol. Organ., Geneva, Switzerland.

Solomon, S., P. J. Crutzen, and R. G. Roble (1982), Photochemical coupling between the thermosphere and the lower atmosphere: 1. Odd nitrogen from 50 to $120 \mathrm{~km}$, J. Geophys. Res., 87, 7206-7220, doi:10.1029/ JC087iC09p07206.

Stamper, R., M. Lockwood, M. N. Wild, and T. D. G. Clark (1999), Solar causes of the long-term increase in geomagnetic activity, J. Geophys. Res., 104, 28,325-28,342, doi:10.1029/1999JA900311.

Stephenson, D. B., V. Pavan, and R. Bojariu (2000), Is the North Atlantic Oscillation a random walk?, Int. J. Climatol., 20, 1-18, doi:10.1002/ (SICI)1097-0088(200001)20:1<1::AID-JOC456>3.0.CO;2-P.

Svensmark, H., and E. Friis-Christensen (1997), Variation of cosmic ray flux and global cloud coverage-A missing link in solar-climate relationships, J. Atmos. Sol. Terr. Phys., 59, 1225-1232, doi:10.1016/S13646826(97)00001-1.

Svensmark, H., T. Bondo, and J. Svensmark (2009), Cosmic ray decreases affect atmospheric aerosols and clouds, Geophys. Res. Lett., 36, L15101, doi:10.1029/2009GL038429.

Swartzman, G., C. H. Huang, and S. Kaluzny (1992), Spatial analysis of Bering Sea groundfish survey data using generalized additive models, Can. J. Fish. Aquat. Sci., 49, 1366-1378, doi:10.1139/f92-152.

Taubenheim, J. (1989), An easy procedure for detecting a discontinuity in a digital time series, Z. Meteorol., 39(6), 344-347.

Thejll, P., B. Christiansen, and H. Gleisner (2003), On correlations between the North Atlantic Oscillation, geopotential heights, and geomagnetic activity, Geophys. Res. Lett., 30(6), 1347, doi:10.1029/2002GL016598.
Thompson, D. W. J., J. M. Wallace, J. J. Kennedy, and P. D. Jones (2010) An abrupt drop in Northern Hemisphere sea surface temperature around 1970, Nature, 467, 444-447, doi:10.1038/nature09394.

Tinsley, B. A., G. B. Burns, and L. Zhou (2007), The role of the global electric circuit in solar and internal forcing of clouds and climate, $A d v$. Space Res., 40, 1126-1139, doi:10.1016/j.asr.2007.01.071.

Underwood, F. M. (2009), Describing long-term trends in precipitation using generalized additive models, J. Hydrol., 364, 285-297, doi:10.1016/j.jhydrol.2008.11.003.

Wood, S. N. (2006), Generalized Additive Models: An Introduction With R, Chapman and Hall, Boca Raton, Fla.

Wood, S. N. (2008), Fast stable direct fitting and smoothness selection for generalized additive models, J. R. Stat. Soc., Ser. B, 70, 495-518, doi:10.1111/j.1467-9868.2007.00646.x.

Woollings, T., A. Hannachi, B. Hoskins, and A. Turner (2010a), A regime view of the North Atlantic Oscillation and its response to anthropogenic forcing, J. Clim., 23, 1291-1307, doi:10.1175/2009JCLI3087.1.

Woollings, T., M. Lockwood, G. Masato, C. Bell, and L. Gray (2010b), Enhanced signature of solar variability in Eurasian winter climate, Geophys. Res. Lett., 37, L20805, doi:10.1029/2010GL044601.

B. Bates, CSIRO Climate Adaptation Flagship, CSIRO Marine and Atmospheric Research, Wembley, WA 6913, Australia. (bryson.bates@ csiro.au)

M. A. Clilverd, M. J. Jarvis, and H. Lu, British Antarctic Survey, High Cross, Madingley Road, Cambridge CB3 0ET, UK. (macl@bas.ac.uk; mjja@bas.ac.uk; hlu@bas.ac.uk)

Y. Li, CSIRO Climate Adaptation Flagship, CSIRO Mathematics, Informatics and Statistics, Wembley, WA 6913, Australia. (yun.li@csiro.au) 